\title{
Exponential Attractors for the 3D Fractional-order Bardina Turbulence Model With Memory and Horizontal Filtering
}

\author{
Michele Annese ${ }^{1} \cdot$ Luca Bisconti $^{2}$ (D) Davide Catania ${ }^{3}$ \\ Dedicated to the memory of Professor Russell Allan Johnson
}

Received: 30 June 2020 / Revised: 29 September 2020 / Accepted: 20 December 2020 /

Published online: 6 February 2021

(c) The Author(s) 2021

\begin{abstract}
We consider the 3D simplified Bardina turbulence model with horizontal filtering, fractional dissipation, and the presence of a memory term incorporating hereditary effects. We analyze the regularity properties and the dissipative nature of the considered system and, in our main result, we show the existence of a global exponential attractor in a suitable phase space.
\end{abstract}

Keywords Simplified Bardina turbulence model - Memory kernels · Anisotropic filters · Dissipation · Exponential attractor

Mathematics Subject Classification 35B40 - 35B65 - 35Q30 - 37L30 - 45K05 - 76D05 . 76F65 - 76D03

\section{Introduction}

Incompressible fluids with constant density are described by the Navier-Stokes equations

$$
\begin{aligned}
& \partial_{t} \boldsymbol{u}+\nabla \cdot(\boldsymbol{u} \otimes \boldsymbol{u})-v \Delta \boldsymbol{u}+\nabla \pi=\boldsymbol{f}, \\
& \nabla \cdot \boldsymbol{u}=0,
\end{aligned}
$$

\footnotetext{
Luca Bisconti

luca.bisconti@unifi.it

Michele Annese

m.annese@unibs.it

Davide Catania

davide.catania@unibs.it
}

1 Università degli Studi di Brescia, Sezione Matematica (DICATAM), Via Valotti 9, I-25133 Brescia, Italia

2 Università degli Studi di Firenze, Dipartimento di Matematica e Informatica “U. Dini”, Via S. Marta 3, I-50139 Firenze, Italia

3 Università eCampus, Facoltà di Ingegneria, Via Isimbardi 10, I-22060 Novedrate, CO, Italia 
supplemented with initial and boundary conditions, where $\boldsymbol{u}(t, \boldsymbol{x})=\left(u_{1}, u_{2}, u_{3}\right)(t, \boldsymbol{x})$ is the velocity field, $\pi(t, \boldsymbol{x})$ denotes the pressure, $\boldsymbol{f}(t, \boldsymbol{x})=\left(f_{1}, f_{2}, f_{3}\right)(t, \boldsymbol{x})$ is the external force, and $v>0$ represents the kinematic viscosity.

In the recent years, the so called " $\alpha$-models" have been proposed to perform numerical simulations of the 3-dimensional fluid Eqs. (1)-(2).

These models are based on a filtering obtained through the application of the inverse of the Helmholtz operator

$$
A=I-\alpha^{2} \Delta,
$$

where $\alpha>0$ is interpreted as a spatial filtering scale.

In this paper, we are concerned with a regularized model for the 3D Navier-Stokes equations derived by the introduction of a suitable horizontal (anisotropic) differential filter and we prove the existence of a global attractor for the corresponding time-shift dynamical system in path-space. Let us consider

$$
\begin{array}{rr}
\boldsymbol{x}=\left(x_{1}, x_{2}, x_{3}\right), & \boldsymbol{x}_{\mathrm{h}}=\left(x_{1}, x_{2}\right), \\
\partial_{j}=\partial_{x_{j}}, \quad \Delta_{\mathrm{h}}=\partial_{1}^{2}+\partial_{2}^{2}, \quad \nabla_{\mathrm{h}}=\left(\partial_{1}, \partial_{2}\right),
\end{array}
$$

where "h" stays for "horizontal" and, instead of choosing the filter given by (3), we take into account the following anisotropic horizontal filter given by (see [5])

$$
A_{\mathrm{h}}=I-\alpha^{2} \Delta_{\mathrm{h}} \quad \text { with } \alpha>0 .
$$

As discussed in $[2,23,27]$, from the point of view of the numerical simulations, this filter is less memory consuming with respect to the standard isotropic one.

The idea behind anisotropic differential filters can be traced back to the approach used by Germano [23]. Recently, the Large Eddy Simulation (LES) community has manifested interest in models involving such a kind of filtering (e.g, $[1,2,4,10,18,20,33])$ and the connection with the family of $\alpha$-models has been highlighted and investigated by Berselli in [5]: exploiting the smoothing provided by the horizontal filtering (4), the author proved global existence and uniqueness of a proper class of weak solutions to the considered regularized model (see the system of Eqs. (6)-(7) below). Again, motivated by [5], in [8,9] a considerable mathematical support to the well-posedness of initial-boundary value problems, in suitable anisotropic Sobolev spaces, to the 3D Boussinesq equations with horizontal filter for turbulent flows is given.

In the sequel, we consider the domain

$$
D=\left\{\boldsymbol{x}=\left(x_{1}, x_{2}, x_{3}\right) \in \mathbb{R}^{3}:-\pi L<x_{1}, x_{2}, x_{3}<\pi L\right\},
$$

$L>0$, with $2 \pi L$ periodicity with respect to $\boldsymbol{x}=\left(x_{1}, x_{2}, x_{3}\right)$, i.e. we are considering a 3D-torus (see Remark 1.1 for motivations on such a choice).

Moreover, in what follows we assume the presence, in (1), of the fractional-order dissipative term $v \Lambda^{2 \beta} \boldsymbol{u}, \Lambda=(-\Delta)^{1 / 2}, 3 / 4 \leq \beta<1$ (see (68) and what follows concerning this assumption), in place of $-v \Delta \boldsymbol{u}$. Although this is a situation of reduced regularity, compared to that of the standard laplacian, the presence of this fractional dissipation allows us to study long-range diffusive behaviors.

Set $\boldsymbol{w}=\overline{\boldsymbol{u}}^{\mathrm{h}}=A_{\mathrm{h}}^{-1} \boldsymbol{u}$ and $q=\bar{\pi}^{\mathrm{h}}=A_{\mathrm{h}}^{-1} \pi$, so that $\boldsymbol{u}=A_{\mathrm{h}} \boldsymbol{w}$. Then, applying the horizontal filter " $-\mathrm{h}$ " component by component to the various fields and tensor fields in (1)-(2), and solving the interior closure problem by the approximation

$$
\overline{\boldsymbol{u} \otimes \boldsymbol{u}} \mathrm{h} \approx{\overline{\overline{\boldsymbol{u}}^{\mathrm{h}} \otimes \overline{\boldsymbol{u}}^{\mathrm{h}}}}^{\mathrm{h}}=\overline{\boldsymbol{w} \otimes \boldsymbol{w}^{\mathrm{h}}},
$$


we finally get the regularized model

$$
\begin{aligned}
& \partial_{t} \boldsymbol{w}+v \Lambda^{2 \beta} \boldsymbol{w}+\nabla \cdot \overline{(\boldsymbol{w} \otimes \boldsymbol{w}}^{\mathrm{h}}+\nabla q=\overline{\boldsymbol{f}}^{\mathrm{h}}, \\
& \nabla \cdot \boldsymbol{w}=0,
\end{aligned}
$$

and we impose a suitable initial condition $\left.\boldsymbol{w}\right|_{t=0}=\boldsymbol{w}_{0}$.

Inspired by [19] (see also [24]), we introduce in the left-hand side of (6) a memory term of the form

$$
\int_{0}^{\infty} g(s) \boldsymbol{w}(t-s) d s .
$$

The map $g:[0, \infty) \rightarrow \mathbb{R}$, called memory kernel, is assumed to be convex, nonnegative, smooth on $\mathbb{R}^{+}=(0, \infty)$, vanishing at infinity and satisfying the further condition

$$
\int_{0}^{\infty} g(s) d s=1 .
$$

The considered model reads as follows:

$$
\begin{aligned}
& \partial_{t} \boldsymbol{w}+v \Lambda^{2 \beta} \boldsymbol{w}+\int_{0}^{\infty} g(s) \boldsymbol{w}(t-s) d s+\nabla \cdot \overline{(\boldsymbol{w} \otimes \boldsymbol{w}}^{\mathrm{h}}+\nabla q=\overline{\boldsymbol{f}}^{\mathrm{h}}, \\
& \nabla \cdot \boldsymbol{w}=0 .
\end{aligned}
$$

The presence of this additional integral term in (9) allows the system to account for memory effects in the fluid. As described in [19,21], the nature of this term owes its origins to the constitutive relations characterizing some families of polymers and geological materials and, in this case, distributed relaxation effects are described through a distributed delay.

System (9)-(10) is supplemented with the initial conditions

$$
\boldsymbol{w}(0)=\boldsymbol{w}_{0} \text { and }\left.\boldsymbol{w}(-s)\right|_{s>0}=\boldsymbol{\varphi}_{0}(s),
$$

where $w_{0}$ and $\varphi_{0}(\cdot)$ are assigned data. Here, $w_{0}$ and $\varphi_{0}(\cdot)$ denote, respectively, the initial velocity field and the past history of the velocity, which is defined for almost every $s>0$.

Due to the nature of the used horizontal filter, a different scheme, with respect to the one used in [19], is needed to carry out our analysis. In fact, despite the smoothing created by the horizontal filter, the regularity gained by the system, and therefore by the weak solutions, does not guarantee neither a direct use of the standard Sobolev inequalities nor the validity of compact embeddings, which are standard tools to prove dissipation and the existence of an absorbing set for the dynamical system associated with the considered system of PDEs. In order to recover, at least partially, these properties and to be able to exploit their consequences, our estimates make explicit use of a splitting of both vector fields and differential operators into horizontal and vertical components (see, e.g., [5,13]).

Moreover, the presence of the memory term makes the situation even more intricate and this fact prevents us to obtain directly energy inequalities. Thus, before applying the just mentioned splitting technique, we rewrite the system in an equivalent but handier way [see the model given by (28)-(30] below).

In this paper we prove existence, uniqueness and regularity for this model. Also, after proving suitable energy decay for the considered problem, estimating the related decay rate, in our main result (Theorem 7.2) we show the existence of a global exponential attractor for the corresponding dynamical system. 
Remark 1.1 A natural domain for the horizontal filter $A_{\mathrm{h}}$ would be

$$
D_{\mathrm{h}}=\left\{\boldsymbol{x}=\left(x_{1}, x_{2}, x_{3}\right) \in \mathbb{R}^{3}:-\pi L<x_{1}, x_{2}<\pi L,-d<x_{3}<d\right\},
$$

$L>0$, with $2 \pi L$ periodicity with respect to $x_{\mathrm{h}}:=\left(x_{1}, x_{2}\right)$, and homogeneous Dirichlet boundary conditions on

$$
\Gamma=\left\{\boldsymbol{x}=\left(x_{1}, x_{2}, x_{3}\right) \in \mathbb{R}^{3}:-\pi L<x_{1}, x_{2}<\pi L, x_{3}= \pm d\right\} .
$$

Actually, another significant advantage in choosing $A_{\mathrm{h}}$ instead of the isotropic operator $A$ is that there is no need to introduce artificial boundary conditions for the Helmholtz operator.

However, such a choice makes problematic to properly define the fractional-order operator $\Lambda^{2 \beta}$ so that it might enjoy all the properties satisfied when defined in a fully periodic setting. A possible way to circumvent this problem is to consider in the space domain $D_{\mathrm{h}}$, with the aforementioned boundary conditions, the fractional anisotropic filter $A_{\mathrm{h}}^{\beta}=I+\alpha^{2 \beta} \Lambda_{\mathrm{h}}{ }^{2 \beta}$, $\Lambda_{\mathrm{h}}=\left(-\Delta_{\mathrm{h}}\right)^{1 / 2}$, with $0<\beta<1$ (see, e.g., $[5,6,12,14]$ ) coupled with the stronger dissipation term $-v \Delta \boldsymbol{w}$, i.e. a full Laplacian appears instead of $v \Lambda^{2 \beta} \boldsymbol{w}$ in (6) (and also in (9)). The case $\beta=1$ is more standard and it will not be considered here. Proceeding as before, this yields the equations

$$
\partial_{t} \boldsymbol{w}-v \Delta \boldsymbol{w}+\int_{0}^{\infty} g(s) \boldsymbol{w}(t-s) d s+\nabla \cdot \overline{(\boldsymbol{w} \otimes \boldsymbol{w}}^{\mathrm{h}}+\nabla q=\overline{\boldsymbol{f}}^{\mathrm{h}}, \quad \nabla \cdot \boldsymbol{w}=0,
$$

instead of (9), where now $\overline{(\cdot)^{\mathrm{h}}}=\left(A_{\mathrm{h}}^{\beta}\right)^{-1}(\cdot)$.

It is possible to verify that the results that we obtain in this paper can be achieved also for the above model, following the same approach. Actually, some parts of the proof can be even simplified and become quite standard. In order to give a more precise idea about this point, apply $A_{\mathrm{h}}^{\beta}$ term by term to (13), to get

$$
\begin{aligned}
& \partial_{t} A_{\mathrm{h}}^{\beta} \boldsymbol{w}+v \Delta A_{\mathrm{h}}^{\beta} \boldsymbol{w}+\int_{0}^{\infty} g(s) A_{\mathrm{h}}^{\beta} \boldsymbol{w}(t-s) d s+(\boldsymbol{w} \cdot \nabla) \boldsymbol{w}+\nabla \pi=\boldsymbol{f}, \\
& \nabla \cdot \boldsymbol{w}=0 .
\end{aligned}
$$

Assuming to have at disposal sufficient regularity to test and manipulate Eq. (14) against $\boldsymbol{w}$ in $\mathrm{L}^{2}\left(D_{\mathrm{h}}\right)$, after an integration by parts we reach

$$
\begin{aligned}
& \frac{1}{2} \frac{d}{d t}\left(\|\boldsymbol{w}\|_{\mathrm{L}^{2}}^{2}+\alpha^{2 \beta}\left\|\Lambda_{\mathrm{h}}^{\beta} \boldsymbol{w}\right\|_{\mathrm{L}^{2}}^{2}\right)+v\left(\left\|\Lambda_{\mathrm{h}}^{\beta} \boldsymbol{w}\right\|_{\mathrm{L}^{2}}^{2}+\alpha^{2 \beta}\left\|\Lambda_{\mathrm{h}}^{\beta} \nabla \boldsymbol{w}\right\|_{\mathrm{L}^{2}}^{2}\right) \\
& \quad+\int_{0}^{\infty} g(s)(\boldsymbol{w}(t-s), \boldsymbol{w}(t))_{\mathrm{L}^{2}} d s \\
& \quad+\alpha^{2 \beta} \int_{0}^{\infty} g(s)\left(\Lambda_{\mathrm{h}}^{\beta} \boldsymbol{w}(t-s), \Lambda_{\mathrm{h}}^{\beta} \boldsymbol{w}(t)\right)_{\mathrm{L}^{2}} d s=(\boldsymbol{f}, \boldsymbol{w})_{\mathrm{L}^{2}},
\end{aligned}
$$

with $\|\cdot\|_{\mathrm{L}^{2}}$ and $(\cdot, \cdot)_{\mathrm{L}^{2}}$, respectively, the norm and the scalar product in $\mathrm{L}^{2}\left(D_{\mathrm{h}}\right)$. Essentially, the presence of the term $v \alpha^{2 \beta}\left\|\Lambda_{\mathrm{h}}^{\beta} \nabla \boldsymbol{w}\right\|_{\mathrm{L}^{2}}^{2}$ in the left-hand side of the above relation, allows us to recover existence, uniqueness, well-posedness and regularity results adapting, to this case, the calculations in Sections 3-to-5 performed in the fully periodic setting. Thus, we judge more interesting the mathematical tools utilized for the proofs when the weaker dissipation term $v \Lambda^{2 \beta} \boldsymbol{w}$ in (9), i.e. $v \Lambda^{2 \beta} \boldsymbol{u}$ in (1), is assumed (and produces $v \alpha^{2}\left\|\Lambda^{\beta} \nabla_{\mathrm{h}} \boldsymbol{w}\right\|_{\mathrm{L}^{2}}^{2}$, after applying $A_{\mathrm{h}}$ to (9) and performing the $\mathrm{L}^{2}$-test); for this reason, in what follows, we will consider the Eqs. (9)-(10) in the periodic context. 
The proposed scheme for our study is as follows.

-Part 1 In Sect. 2 we introduce the notation, some basic facts and preliminary results. In particular, after properly rewriting system (9)-(10) [see Eqs. (28)-(30) below].

-Part 2 Existence of suitable class of weak solutions is established in Sect. 3. In Sect. 3.1, we provide a priori estimates for the considered system in suitable lower order norms. Higher order energy estimates, which are needed to prove the existence of an exponential attractor, are provided in subsequent sections. Section 4 is devoted to the study of continuous dependence and uniqueness of weak solutions.

-Part 3 Then, in Sects. 5 and 6 , we use the dissipative properties of the system to analyze energy decay and dynamics. In so doing we prove the existence of a bounded absorbing set. Finally, in Sect. 7 we show the existence of the global exponential attractor for the dynamical system associated to Eqs. (9)-(10).

\section{Preliminaries on the Regularized Model, Basic Facts and Notation}

We introduce the following function spaces:

$$
\begin{aligned}
& \mathrm{L}^{2}(D)=\left\{\phi: D \rightarrow \mathbb{R} \text { measurable, } 2 \pi L \text { periodic in } \boldsymbol{x}, \int_{D}|\phi|^{2} \mathrm{~d} \boldsymbol{x}<+\infty\right\}, \\
& \mathrm{L}_{0}^{2}(D)=\left\{\phi \in \mathrm{L}^{2}(D) \text { with zero mean with respect to } x\right\}, \\
& \mathrm{H}=\left\{\boldsymbol{\phi} \in\left(\mathrm{L}_{0}^{2}(D)\right)^{3}: \nabla \cdot \boldsymbol{\phi}=0 \text { in } D\right\},
\end{aligned}
$$

all with $\mathrm{L}^{2}$ norm denoted by $\|\cdot\|$, and scalar product $(\cdot, \cdot)$ in $\mathrm{L}^{2}$. Moreover, we set

$$
\begin{aligned}
& \mathrm{V}=\left\{\boldsymbol{\phi} \in \mathrm{H}: \nabla \boldsymbol{\phi} \in\left(\mathrm{L}^{2}(D)\right)^{9}\right\}, \\
& \mathrm{V}_{\mathrm{h}}=\left\{\boldsymbol{\phi} \in \mathrm{H}: \nabla_{\mathrm{h}} \boldsymbol{\phi} \in\left(\mathrm{L}^{2}(D)\right)^{6}\right\} .
\end{aligned}
$$

The space $V_{h}$ is endowed with the inner product

$$
\langle\boldsymbol{u}, \boldsymbol{v}\rangle_{\mathrm{V}_{\mathrm{h}}}=(\boldsymbol{u}, \boldsymbol{v})+\alpha^{2}\left(\nabla_{\mathrm{h}} \boldsymbol{u}, \nabla_{\mathrm{h}} \boldsymbol{v}\right),
$$

where $\|\boldsymbol{u}\|_{\mathrm{V}_{\mathrm{h}}}^{2}=\|\boldsymbol{u}\|^{2}+\alpha^{2}\left\|\nabla_{\mathrm{h}} \boldsymbol{u}\right\|^{2}$.

Further, for any $0<\beta<1$, we define

$$
\begin{aligned}
& \mathrm{H}^{\beta}=\left\{\boldsymbol{\phi} \in \mathrm{H}: \Lambda^{\beta} \boldsymbol{\phi} \in \mathrm{L}^{2}(D)^{3}\right\}, \\
& \mathrm{H}_{\mathrm{h}}^{1+\beta}=\left\{\boldsymbol{\phi} \in \mathrm{V}_{\mathrm{h}}: \Lambda^{\beta} \boldsymbol{\phi} \in \mathrm{V}_{\mathrm{h}}\right\}, \\
& \mathrm{H}_{\mathrm{h}}^{1+2 \beta}=\left\{\boldsymbol{\phi} \in \mathrm{H}_{\mathrm{h}}^{1+\beta}: \Lambda^{2 \beta} \boldsymbol{\phi} \in \mathrm{V}_{\mathrm{h}}\right\}
\end{aligned}
$$

with norms, respectively,

$$
\begin{aligned}
& \|\boldsymbol{\phi}\|_{\mathrm{H}^{\beta}}^{2}=\|\boldsymbol{\phi}\|^{2}+\left\|\Lambda^{\beta} \boldsymbol{\phi}\right\|^{2}, \\
& \|\boldsymbol{\phi}\|_{\mathrm{H}_{\mathrm{h}}^{1+\beta}}^{2+\beta}=\|\boldsymbol{\phi}\|_{\mathrm{V}_{\mathrm{h}}}^{2}+\left\|\Lambda^{\beta} \boldsymbol{\phi}\right\|_{\mathrm{V}_{\mathrm{h}}}^{2}, \\
& \|\boldsymbol{\phi}\|_{\mathrm{H}_{\mathrm{h}}^{1+2 \beta}}^{2}=\|\boldsymbol{\phi}\|_{\mathrm{H}_{\mathrm{h}}^{1+\beta}}^{2}+\left\|\Lambda^{2 \beta} \boldsymbol{\phi}\right\|_{\mathrm{V}_{\mathrm{h}}}^{2} .
\end{aligned}
$$

In the sequel, in order to keep the notation compact, we omit the superscript indicating the dimension of the considered spaces, reintroducing it only when it is strictly required by the context. 
In the sequel (especially to get estimates in $\mathrm{H}^{s}$, where $s$ is not integer) we shall also use some commutator-type estimates as the one in the following lemma concerning the operator $\Lambda^{s}, s \in \mathbb{R}^{+}$(see, e.g., [25,26,34], see also [7,36]), with $\Lambda=(-\Delta)^{1 / 2}$.

Lemma 2.1 For $s>0$ and $1<r \leq \infty$, and for smooth enough $u$ and $v$, we get

$$
\left\|\Lambda^{s}(u v)\right\|_{\mathrm{L}^{r}} \leq c\left(\|u\|_{\mathrm{L}^{p_{1}}}\left\|\Lambda^{s} v\right\|_{\mathrm{L}^{q_{1}}}+\|v\|_{\mathrm{L}^{\mathrm{p} 2}}\left\|\Lambda^{s} u\right\|_{\mathrm{L}^{q_{2}}}\right),
$$

where $1 / r=1 / p_{1}+1 / q_{1}=1 / p_{2}+1 / q_{2}$ and $c$ is a suitable positive constant.

We will make use of the following result about product-laws in Sobolev spaces (see $[17,32])$

Lemma 2.2 Let $s_{0}, s_{1}, s_{2} \in \mathbb{R}$. The product estimate

$$
\|u v\|_{\mathrm{H}^{-s_{0}}} \leq c\|u\|_{\mathrm{H}^{s_{1}}}\|v\|_{\mathrm{H}^{s_{2}}}
$$

holds, provided that

$$
\begin{aligned}
& s_{0}+s_{1}+s_{2} \geq \frac{n}{2}, \text { where } n \text { is the space dimension, } \\
& s_{0}+s_{1} \geq 0, \\
& s_{0}+s_{2} \geq 0, \\
& s_{1}+s_{2} \geq 0 .
\end{aligned}
$$

If in (18) the equality sign holds, inequalities (19)-(21) must be strict.

\subsection{Regularized Model With Memory}

To get the considered model, we apply the operator $A_{\mathrm{h}}$ defined in (4), term by term to (9)-(10) and, following the scheme proposed in [19], we finally reach

$$
\begin{aligned}
& \partial_{t} A_{\mathrm{h}} \boldsymbol{w}+v \Lambda^{2 \beta} A_{\mathrm{h}} \boldsymbol{w}+\int_{0}^{\infty} g(s) A_{\mathrm{h}} \boldsymbol{w}(t-s) d s+(\boldsymbol{w} \cdot \nabla) \boldsymbol{w}+\nabla \pi=\boldsymbol{f}, \\
& \nabla \cdot \boldsymbol{w}=0
\end{aligned}
$$

where $g$ has been introduced in (8). This is the simplified Bardina model with horizontal filter, memory and fractional viscosity and we supply this problem with periodic boundary conditions. Thus, system (23)-(24) can be thought on the $3 \mathrm{D}$-torus $\mathbb{T}^{3}:=\mathbb{R}^{3} / 2 \pi L \mathbb{Z}^{3}$.

Before starting our analysis, we give further notation and hypotheses to plug system (23)(24) in a suitable framework. We introduce the following $\mathrm{L}^{2}$-weighted Hilbert space on $\mathbb{R}^{+}$, i.e.

$$
\mathcal{M}_{\mathrm{h}}^{1}=\mathrm{L}_{\mu}^{2}\left(\mathbb{R}^{+}, \mathrm{V}_{\mathrm{h}}\right)
$$

with inner product

$$
\langle\eta, \xi\rangle_{\mathcal{M}_{\mathrm{h}}^{1}}=\int_{0}^{\infty} \mu(s)\langle\eta(s), \xi(s)\rangle_{V_{\mathrm{h}}} d s
$$

with $(\cdot, \cdot)$ the $\mathrm{L}^{2}$-inner product and

$$
\|\eta\|_{\mathcal{M}_{\mathrm{h}}^{1}}=\left(\int_{0}^{\infty} \mu(s)\|\eta(s)\|_{\mathrm{V}_{\mathrm{h}}}^{2} d s\right)^{\frac{1}{2}}=\left(\int_{0}^{\infty} \mu(s)\left(\|\eta(s)\|^{2}+\alpha^{2}\left\|\nabla_{\mathrm{h}} \eta(s)\right\|^{2}\right) d s\right)^{\frac{1}{2}} .
$$


Here, we assume $\mu$ nonnegative, absolutely continuous, decreasing (hence $\mu^{\prime} \leq 0$ a. e. on $\mathbb{R}^{+}$), and summable on $\mathbb{R}^{+}$with total mass

$$
\kappa=\int_{0}^{\infty} \mu(s) d s>0
$$

Further conditions on the kernel $\mu$ will be provided at the end of this subsection.

The infinitesimal generator of the right-translation semigroup on $\mathcal{M}_{\mathrm{h}}^{1}$ is given by $T \eta=$ $-\partial_{s} \eta$, with domain

$$
D(T)=\left\{\eta \in \mathcal{M}_{\mathrm{h}}^{1}: \partial_{s} \eta \in \mathcal{M}_{\mathrm{h}}^{1}, \eta(0)=0\right\} .
$$

Here, $\partial_{s} \eta$ is the distributional derivative of $\eta(s)$ with respect to the internal variable s. Finally, we define the extended memory space $\mathcal{H}_{\mathrm{h}}^{1}=\mathrm{V}_{\mathrm{h}} \times \mathcal{M}_{\mathrm{h}}^{1}$ endowed with the product norm

$$
\|(\boldsymbol{w}, \eta)\|_{\mathcal{H}_{\mathrm{h}}^{1}}^{2}=\|\boldsymbol{w}\|^{2}+\alpha^{2}\left\|\nabla_{\mathrm{h}} \boldsymbol{w}\right\|^{2}+\|\eta\|_{\mathcal{M}_{\mathrm{h}}^{1}}^{2}
$$

We also use more regular spaces: To this end we define

$$
\mathcal{M}_{\mathrm{h}}^{1+\beta}=\mathrm{L}_{\mu}^{2}\left(\mathbb{R}^{+} ; \mathrm{H}_{\mathrm{h}}^{1+\beta}\right),
$$

with inner product analogous to that of $\mathcal{M}_{\mathrm{h}}$, and norm given by

$$
\|\eta\|_{\mathcal{M}_{\mathrm{h}}^{1+\beta}}=\left(\int_{0}^{\infty} \mu(s)\|\eta(s)\|_{\mathrm{H}_{\mathrm{h}}^{1+\beta}}^{2} d s\right)^{\frac{1}{2}} .
$$

The corresponding higher order extended memory space $\mathcal{H}_{\mathrm{h}}^{1+\beta}=\mathrm{H}_{\mathrm{h}}^{1+\beta} \times \mathcal{M}_{\mathrm{h}}^{1+\beta}$ is endowed with norm

$$
\|(\boldsymbol{w}, \eta)\|_{\mathcal{H}_{\mathrm{h}}^{1+\beta}}^{2}=\|\boldsymbol{w}\|_{\mathrm{H}_{\mathrm{h}}^{1+\beta}}^{2}+\|\eta\|_{\mathcal{M}_{\mathrm{h}}^{1+\beta}}^{2}
$$

Again, in the sequel we will also consider $\mathcal{M}_{\mathrm{h}}^{1+2 \beta}=\mathrm{L}_{\mu}^{2}\left(\mathbb{R}^{+} ; \mathrm{H}_{\mathrm{h}}^{1+2 \beta}\right)$ and the extended memory space $\mathcal{H}_{\mathrm{h}}^{1+2 \beta}=\mathrm{H}_{\mathrm{h}}^{1+2 \beta} \times \mathcal{M}_{\mathrm{h}}^{1+2 \beta}$.

Following [19] (see also [24]), we introduce the past history variable

$$
\eta^{t}(s)=\int_{0}^{s} w(t-\sigma) d \sigma=\int_{t-s}^{t} w(\ell) d \ell, \quad s \geq 0, t>s,
$$

which satisfies the following differential identity

$$
\partial_{t} \eta^{t}(s)=-\partial_{s} \eta^{t}(s)+\boldsymbol{w}(t)
$$

Now, consider system (23)-(24) coupled with the previous equation. Using the operator $T=-\partial_{s}$, an integration by parts in $d s$ leads to an equivalent differential problem in the variables $\boldsymbol{w}=\boldsymbol{w}(t)$ and $\eta=\eta^{t}(\cdot)$, i.e. 


$$
\begin{aligned}
& \partial_{t} A_{\mathrm{h}} \boldsymbol{w}+v \Lambda^{2 \beta} A_{\mathrm{h}} \boldsymbol{w}+\int_{0}^{\infty} \mu(s) A_{\mathrm{h}} \eta(s) d s+(\boldsymbol{w} \cdot \nabla) \boldsymbol{w}+\nabla \pi=\boldsymbol{f}, \\
& \partial_{t} \eta=T \eta+\boldsymbol{w}, \\
& \nabla \cdot \boldsymbol{w}=0,
\end{aligned}
$$

where $\mu=-g^{\prime}$ and the system is considered on the $3 D$-torus $\mathbb{T}^{3}$. In what follows, we assume $g$ such that $\mu \in C^{1}\left(\mathbb{R}^{+}\right) \cap \mathrm{L}^{1}\left(\mathbb{R}^{+}\right), \mu(s) \geq 0$ and $\mu^{\prime}(s) \leq 0$ for every $s \in \mathbb{R}^{+}$. We also assume (see [19]) the condition

$$
\mu^{\prime}(s)+\delta \mu(s) \leq 0
$$

for some $\delta>0$ and almost every $s>0$.

For the system (28)-(30), but also for the case corresponding to (23)-(24), the initial conditions (11) can be rewritten as follows:

$$
\boldsymbol{w}(0)=\boldsymbol{w}_{0} \text { and } \eta^{0}=\eta_{0},
$$

and

$$
\eta_{0}(s):=\int_{0}^{s} \varphi_{0}(\tau) d \tau .
$$

We will provide a precise definition of weak solution for system (23)-(24) at the beginning of Sect. 3.

\section{Existence}

Let us introduce the definition of "regular weak solution".

Definition 3.1 Given $U_{0}=\left(\boldsymbol{w}_{0}, \eta_{0}\right) \in \mathcal{H}_{\mathrm{h}}^{1}$, a function $U=(\boldsymbol{w}, \eta) \in C\left([0, \infty), \mathcal{H}_{\mathrm{h}}^{1}\right)$, with $w \in \mathrm{L}^{\infty}\left(0, T ; \mathrm{V}_{\mathrm{h}}\right) \cap L^{2}\left(0, T ; \mathrm{H}_{\mathrm{h}}^{1+\beta}\right), w_{3} \in \mathrm{L}^{\infty}(0, T ; \mathrm{V}) \cap \mathrm{L}^{2}\left(0, T ; \mathrm{H}^{1+\beta}\right), \eta \in$ $\mathrm{L}^{\infty}\left(0, T ; \mathcal{M}_{\mathrm{h}}^{1}\right)$, and $A_{\mathrm{h}} \partial_{t} w \in \mathrm{L}^{2}\left(0, \tau ; \mathrm{H}^{-\beta}\right)$ for every $\tau>0$, is a regular weak solution of (28)-(30) with initial datum $U(0)=\left(\boldsymbol{w}(0), \eta^{0}\right)=U_{0}$ if for every test function $\boldsymbol{v} \in \mathrm{H}_{\mathrm{h}}^{1+\beta}$ and almost every $t>0$ we have that

$$
\begin{aligned}
& \left(\partial_{t} \boldsymbol{w}, \boldsymbol{v}\right)+\alpha^{2}\left(\nabla_{\mathrm{h}} \partial_{t} \boldsymbol{w}, \nabla_{\mathrm{h}} \boldsymbol{v}\right)+v\left(\Lambda^{\beta} \boldsymbol{w}, \Lambda^{\beta} \boldsymbol{v}\right)+v \alpha^{2}\left(\Lambda^{\beta} \nabla_{\mathrm{h}} \boldsymbol{w}, \Lambda^{\beta} \nabla_{\mathrm{h}} \boldsymbol{v}\right) \\
& \quad+\int_{0}^{\infty} \mu(s)\langle\eta(s), \boldsymbol{v}\rangle_{V_{\mathrm{h}}} d s+((\boldsymbol{w} \cdot \nabla) \boldsymbol{w}, \boldsymbol{v})=(\boldsymbol{f}, \boldsymbol{v}),
\end{aligned}
$$

where $\eta$ is such that

$$
\eta^{t}(s)= \begin{cases}\int_{0}^{s} \boldsymbol{w}(t-\tau) d \tau, & 0<s \leq t, \\ \eta^{0}(s-t)+\int_{0}^{t} \boldsymbol{w}(t-\tau) d \tau, & s>t .\end{cases}
$$

Also, as we will see later (in relation (58) below) that this solution satisfies a suitable energy-like equality instead of only an energy inequality as in the case of the standard Navier-Stokes equations.

Here we proceed formally to derive an energy estimate (also in this case the calculations can be made more rigorous by using a proper Galerkin scheme, see the following section) starting from initial data $\left(w_{0}, \eta_{0}\right) \in \mathcal{H}_{\mathrm{h}}^{1}$. 
Observe that, starting from initial data $\left(\boldsymbol{w}_{0}, \eta_{0}\right) \in \mathcal{H}_{\mathrm{h}}^{1}$, following the classical approach for the Navier-Stokes equations, and using suitable controls on $\boldsymbol{w}, \nabla_{\mathrm{h}} \boldsymbol{w}$ and $\partial_{t} \boldsymbol{w}$ (see relations (46) and (52), below), one can prove the existence of weak solutions analogous to Leray-Hopf solutions (see, e.g., [22]).

Therefore, the existence of weak solutions is carried out by exploiting the energy estimates provided in the next parts of this section, and then passing to the limit in the usual way. We refer to $[16,19]$ for more details on the Galerkin scheme in connection with equations with memory.

In Sect. 5, in order to study the existence of an exponential attractor for the dynamical system associated with (28)-(30), we will be interested in more regular solutions and show, beyond the control at the end of this section, higher order estimates for $\partial_{t} \boldsymbol{w}$.

\subsection{A Priori Estimates}

In the sequel we proceed formally in order to find appropriate energy estimates. As already mentioned, a rigorous proof can be easily obtained by introducing a suitable Galerkin approximation scheme $\left\{\left(\boldsymbol{w}_{k}, \eta_{k}\right)\right\}$ (see, e.g., [22]).

Testing (28) and (29) against $\boldsymbol{w}$ and $\eta$, respectively, we get

$$
\begin{aligned}
& \frac{1}{2} \frac{d}{d t}\left(\|\boldsymbol{w}\|^{2}+\alpha^{2}\left\|\nabla_{\mathrm{h}} \boldsymbol{w}\right\|^{2}\right)+v\left(\left\|\Lambda^{\beta} \boldsymbol{w}\right\|^{2}+\alpha^{2}\left\|\Lambda^{\beta} \nabla_{\mathrm{h}} \boldsymbol{w}\right\|^{2}\right) \\
& \quad+\int_{0}^{\infty} \mu(s)\langle\eta, \boldsymbol{w}\rangle_{V_{\mathrm{h}}} d s=(f, \boldsymbol{w}), \\
& \int_{0}^{\infty} \mu(s)\left\langle\partial_{t} \eta, \eta\right\rangle_{V_{\mathrm{h}}} d s=-\int_{0}^{\infty} \mu(s)\left\langle\partial_{s} \eta, \eta\right\rangle_{V_{\mathrm{h}}} d s+\int_{0}^{\infty} \mu(s)\langle\boldsymbol{w}, \eta\rangle_{V_{\mathrm{h}}} d s,
\end{aligned}
$$

the above second equation can be rewritten as

$$
\frac{1}{2} \frac{d}{d t}\|\eta\|_{\mathcal{M}_{\mathrm{h}}^{1}}^{2}-\int_{0}^{\infty} \mu(s)\langle\eta, \boldsymbol{w}\rangle_{V_{\mathrm{h}}} d s-\langle T \eta, \eta\rangle_{\mathcal{M}_{\mathrm{h}}^{1}}=0
$$

and summing up (33) and (35) we get

$$
\begin{aligned}
& \frac{1}{2} \frac{d}{d t}\left(\|\boldsymbol{w}\|^{2}+\alpha^{2}\left\|\nabla_{\mathrm{h}} \boldsymbol{w}\right\|^{2}+\|\eta\|_{\mathcal{M}_{\mathrm{h}}^{1}}^{2}\right)+v\left(\left\|\Lambda^{\beta} \boldsymbol{w}\right\|^{2}+\alpha^{2}\left\|\Lambda^{\beta} \nabla_{\mathrm{h}} \boldsymbol{w}\right\|^{2}\right) \\
& \quad-\langle T \eta, \eta\rangle_{\mathcal{M}_{\mathrm{h}}^{1}}=(\boldsymbol{f}, \boldsymbol{w}) .
\end{aligned}
$$

To prove that $\langle T \eta, \eta\rangle_{\mathcal{M}_{\mathrm{h}}^{1}} \leq 0$, we can use directly the argument in the proof of [24, Theorem 3.1], and we report it here-adapted to our context—for the sake of completeness. For any $\eta \in D(T)$ [see (26)], we have that

$$
\begin{aligned}
\langle T \eta, \eta\rangle_{\mathcal{M}_{\mathrm{h}}^{1}} & =-\frac{1}{2} \int_{0}^{\infty} \mu(s) \frac{d}{d s}\|\eta(s)\|_{\mathrm{V}_{\mathrm{h}}}^{2} d s \\
& =\frac{1}{2} \lim _{\tau \rightarrow 0}\left(-\mu(1 / \tau)\|\eta(1 / \tau)\|_{\mathrm{V}_{\mathrm{h}}}^{2}+\mu(\tau)\|\eta(\tau)\|_{\mathrm{V}_{\mathrm{h}}}^{2}+\int_{\tau}^{1 / \tau} \mu^{\prime}(s)\|\eta(s)\|_{\mathrm{V}_{\mathrm{h}}}^{2} d s\right) .
\end{aligned}
$$


Now, observing that

$$
\begin{aligned}
\lim _{\tau \rightarrow 0} \mu(\tau)\|\eta(\tau)\|_{\mathrm{V}_{\mathrm{h}}}^{2} & \leq \limsup _{\tau \rightarrow 0} \mu(\tau)\left(\int_{0}^{\tau} \frac{d}{d \ell}\|\eta(\ell)\|_{\mathrm{V}_{\mathrm{h}}} d \ell\right)^{2} \\
& \leq \limsup _{\tau \rightarrow 0}\left(\int_{0}^{\tau} \mu(\ell)^{\frac{1}{2}} \frac{d}{d \ell}\|\eta(\ell)\| \mathrm{v}_{\mathrm{h}} d \ell\right)^{2} \\
& \leq \limsup _{\tau \rightarrow 0} \int_{0}^{\tau} \mu(\ell)\left\|\eta^{\prime}(\ell)\right\|_{\mathrm{V}_{\mathrm{h}}}^{2} d \ell=0 .
\end{aligned}
$$

But the left-hand side of (37) is bounded, and the remaining two terms of the right-hand side are negative. Then, we can conclude that both the integral and the limit exist and are finite. In particular, this implies that the limit equals zero. As a direct consequence, we have that

$$
\langle T \eta, \eta\rangle_{\mathcal{M}_{\mathrm{h}}^{1}}=\int_{0}^{\infty} \mu^{\prime}(s)\|\eta(s)\|_{\mathrm{V}_{\mathrm{h}}}^{2} d s \leq 0 .
$$

Moreover, we also have that (see, e.g., [19] for isotropic flows)

$$
\frac{\delta}{2}\|\eta\|_{\mathcal{M}_{\mathrm{h}}^{1}}^{2} \leq-\langle T \eta, \eta\rangle_{\mathcal{M}_{\mathrm{h}}^{1}}
$$

with $\delta>0$ suitable constant.

Relation (36) along with (38)-(39) produces a first energy estimate for the system (28)(30) in $\mathcal{M}_{\mathrm{h}}^{1}$. However, to get further a priori estimates, needed in order to prove existence, some additional regularity is required in the considered model.

Remark 3.2 From (36), given an initial datum $\left(\boldsymbol{w}_{0}, \eta_{0}\right) \in \mathcal{H}_{\mathrm{h}}^{1}$, the corresponding energy at time $t \geq 0$, reads

$$
\mathcal{E}(t)=\frac{1}{2}\left(\|\boldsymbol{w}\|^{2}+\alpha^{2}\left\|\nabla_{\mathrm{h}} \boldsymbol{w}\right\|^{2}+\|\eta\|_{\mathcal{M}_{\mathrm{h}}^{1}}^{2}\right) .
$$

As a further step in order to prove the existence, we now consider relation (36), and using

$$
(\boldsymbol{f}, \boldsymbol{w}) \leq \varepsilon\left\|\Lambda^{\beta} \boldsymbol{w}\right\|^{2}+\frac{c}{\varepsilon}\left\|\Lambda^{-\beta} \boldsymbol{f}\right\|^{2},
$$

along with (39), we get

$$
\begin{aligned}
& \frac{1}{2} \frac{d}{d t}\left(\|\boldsymbol{w}\|^{2}+\alpha^{2}\left\|\nabla_{\mathrm{h}} \boldsymbol{w}\right\|^{2}+\|\eta\|_{\mathcal{M}_{\mathrm{h}}^{1}}^{2}\right)+(v-\varepsilon)\left(\left\|\Lambda^{\beta} \boldsymbol{w}\right\|^{2}+\alpha^{2}\left\|\nabla_{\mathrm{h}} \Lambda^{\beta} \boldsymbol{w}\right\|^{2}\right)+\frac{\delta}{2}\|\eta\|_{\mathcal{M}_{\mathrm{h}}^{1}}^{2} \\
& \quad \leq \frac{c}{\varepsilon}\left\|\Lambda^{-\beta} \boldsymbol{f}\right\|^{2},
\end{aligned}
$$

and, in particular, we infer

$$
\begin{aligned}
& \frac{1}{2} \frac{d}{d t}\left(\|\boldsymbol{w}\|^{2}+\alpha^{2}\left\|\nabla_{\mathrm{h}} \boldsymbol{w}\right\|^{2}+\|\eta\|_{\mathcal{M}_{\mathrm{h}}^{1}}^{2}\right)+\frac{\hat{v}}{2}\left(\left\|\Lambda^{\beta} \boldsymbol{w}\right\|^{2}+\alpha^{2}\left\|\nabla_{\mathrm{h}} \Lambda^{\beta} \boldsymbol{w}\right\|^{2}+\|\eta\|_{\mathcal{M}_{\mathrm{h}}^{1}}^{2}\right) \\
& \quad \leq \frac{c}{\varepsilon}\left\|\Lambda^{-\beta} \boldsymbol{f}\right\|^{2} .
\end{aligned}
$$

Remark 3.3 Using Hölder's and Gagliardo-Nirenberg's inequalities we have that:

$$
\|\boldsymbol{w}\| \leq c\|\boldsymbol{w}\|_{\mathrm{L}^{p}}, \quad p \geq 2 \text { and }\|\boldsymbol{w}\|_{\mathrm{L}^{p}} \leq C\|\boldsymbol{w}\|^{1-\sigma}\left\|\Lambda^{\beta} \boldsymbol{w}\right\|^{\sigma}
$$

with

$$
0<\frac{3(p-2)}{2 p \beta}=\sigma \leq 1 .
$$


As a consequence of the above control, and assuming $3 / 4 \leq \beta<1$ [see relation (68) below, and its consequences; this provides a lower bound for $\beta$ ], we have that

$$
0<\frac{3(p-2)}{2 p} \leq \frac{3}{4}
$$

which implies, for $2<p \leq 4$, that $\|\boldsymbol{w}\|^{\sigma} \leq c\left\|\Lambda^{\beta} \boldsymbol{w}\right\|^{\sigma}$, and so

$$
\|\boldsymbol{w}\| \leq c\left\|\Lambda^{\beta} \boldsymbol{w}\right\| \text {. }
$$

Then, exploiting (42) along with (44), we reach

$$
\frac{1}{2} \frac{d}{d t}\left(\|\boldsymbol{w}\|^{2}+\alpha^{2}\left\|\nabla_{\mathrm{h}} \boldsymbol{w}\right\|^{2}+\|\eta\|_{\mathcal{M}_{\mathrm{h}}^{1}}^{2}\right)+\frac{\hat{v}}{2}\left(\|\boldsymbol{w}\|^{2}+\alpha^{2}\left\|\nabla_{\mathrm{h}} \boldsymbol{w}\right\|^{2}+\|\eta\|_{\mathcal{M}_{\mathrm{h}}^{1}}^{2}\right) \leq \frac{c}{\varepsilon}\left\|\Lambda^{-\beta} \boldsymbol{f}\right\|^{2},
$$

where $\hat{v}:=c \hat{v}$. Then, setting $Y(t)=\left(\|\boldsymbol{w}\|^{2}+\alpha^{2}\left\|\nabla_{\mathrm{h}} \boldsymbol{w}\right\|^{2}+\|\eta\|_{\mathcal{M}_{\mathrm{h}}^{1}}^{2}\right)(t)$, from the above differential inequality we obtain

$$
\begin{aligned}
Y(t) & \leq Y_{0} e^{-\hat{v} t}+c\left\|\Lambda^{-\beta} \boldsymbol{f}\right\|^{2}\left(1-e^{-\hat{v} t}\right) \\
& \leq Y_{0} e^{-\hat{v} t}+c\left\|\Lambda^{-\beta} \boldsymbol{f}\right\|^{2},
\end{aligned}
$$

that is, the following global estimate (here $f \in \mathrm{L}^{2}(D)$ ):

$$
\begin{aligned}
& \left(\|\boldsymbol{w}\|^{2}+\alpha^{2}\left\|\nabla_{\mathrm{h}} \boldsymbol{w}\right\|^{2}+\|\eta\|_{\mathcal{M}_{\mathrm{h}}^{1}}^{2}\right)(t) \\
& \quad \leq\left(\left\|\boldsymbol{w}_{0}\right\|^{2}+\alpha^{2}\left\|\nabla_{\mathrm{h}} \boldsymbol{w}_{0}\right\|^{2}+\left\|\eta_{0}\right\|_{\mathcal{M}_{\mathrm{h}}^{1}}^{2}\right) e^{-\hat{v} t}+c\left\|\Lambda^{-\beta} \boldsymbol{f}\right\|^{2} .
\end{aligned}
$$

In particular, we have $w \in \mathrm{L}^{\infty}\left(0, \infty ; \mathrm{V}_{\mathrm{h}}\right), w_{3} \in \mathrm{L}^{\infty}\left(0, \infty ; \mathrm{H}^{1}\right)$ and $\eta \in \mathrm{L}^{\infty}\left(0, \infty ; \mathcal{M}_{\mathrm{h}}^{1}\right)$. We also obtain that $\Lambda^{\beta} \boldsymbol{w} \in \mathrm{L}^{2}\left(0, T ; \mathrm{V}_{\mathrm{h}}\right), \Lambda^{\beta} w_{3} \in \mathrm{L}^{2}\left(0, T ; \mathrm{H}^{1}\right), T>0$.

Remark 3.4 If we assume that $f \in \mathrm{L}^{2}\left(0,+\infty ; \mathrm{H}^{-\beta}\right)$, adapting (42) accordingly, then we can obtain that $\Lambda^{\beta} \boldsymbol{w} \in \mathrm{L}^{2}\left(0,+\infty ; \mathrm{V}_{\mathrm{h}}\right), \Lambda^{\beta} w_{3} \in \mathrm{L}^{2}\left(0, \infty ; \mathrm{H}^{1}\right)$. Also, from (46), it follows that $\mathcal{E} \in \mathrm{L}^{1}(0,+\infty)$, where $\mathcal{E}=\mathcal{E}(t)$ was defined in (40).

Remark 3.5 We recall that $U(t)=(\boldsymbol{w}(t), \eta(t))$ and assume $\|U(0)\|_{\mathcal{H}_{\mathrm{h}}^{1}} \leq R$. Integrating (42) in time over $[0, T]$, for any $T>0$, we deduce in particular

$$
\|U(T)\|_{\mathcal{H}_{\mathrm{h}}^{1}}^{2} \leq\|U(0)\|_{\mathcal{H}_{\mathrm{h}}^{1}}^{2}+C T \leq \mathcal{Q}\left((R+T)^{2}\right)
$$

where

$$
\mathcal{Q}(t):=C_{1}(t+C \sqrt{t}), \quad C_{1}>0 \text { large enough. }
$$

Let us notice, in particular, that the function $\mathcal{Q}(t)$ is positive and increasing. Then, integrating (42), we get:

$$
\begin{aligned}
& \left(\|\boldsymbol{w}\|^{2}+\alpha^{2}\left\|\nabla_{\mathrm{h}} \boldsymbol{w}\right\|^{2}+\|\eta\|_{\mathcal{M}_{\mathrm{h}}^{1}}^{2}\right)+\int_{0}^{t}\left[2(v-\varepsilon)\left(\left\|\Lambda^{\beta} \boldsymbol{w}\right\|^{2}+\alpha^{2}\left\|\nabla_{\mathrm{h}} \Lambda^{\beta} \boldsymbol{w}\right\|\right)+\delta\|\eta\|_{\mathcal{M}_{\mathrm{h}}^{1}}^{2}\right] d s \\
& \leq \mathcal{Q}\left((R+t)^{2}\right) .
\end{aligned}
$$

Moreover, using (48), relation(46) can be rewritten as:

$$
\left(\|\boldsymbol{w}\|^{2}+\alpha^{2}\left\|\nabla_{\mathrm{h}} \boldsymbol{w}\right\|^{2}+\|\eta\|_{\mathcal{M}_{\mathrm{h}}^{1}}^{2}\right)(t) \leq \mathcal{Q}\left(\|U(0)\|_{\mathcal{H}_{\mathrm{h}}^{1}}^{2}\right) e^{-\hat{v} t}+\hat{c}\left\|\Lambda^{-\beta} \boldsymbol{f}\right\|^{2},
$$

with $\hat{c}=c / \varepsilon$.

Remark 3.6 Hereafter, $\mathcal{Q}(t)$ will denote any positive increasing function, that can change every time. 


\subsection{Estimate for $w_{t}$}

Let us assume $\left(\boldsymbol{w}_{0}, \eta_{0}\right) \in \mathcal{H}_{\mathrm{h}}^{1}=\mathrm{V}_{\mathrm{h}} \times \mathcal{M}_{\mathrm{h}}^{1}$ and $\boldsymbol{f}=\boldsymbol{f}(x)$. Then testing (28) against $\phi \in \mathrm{H}^{\beta}$, we get

$$
\begin{gathered}
\left|\left(\boldsymbol{w}_{t}+\alpha^{2} \Delta_{\mathrm{h}} \boldsymbol{w}_{t}, \phi\right)\right| \leq c\left(v\left(\left\|\Lambda^{\beta} \boldsymbol{w}\right\|+\alpha^{2}\left\|\nabla_{\mathrm{h}} \Lambda^{\beta} \boldsymbol{w}\right\|\right)+\|\eta\|_{\mathcal{M}_{\mathrm{h}}^{1}}\right. \\
+\|\boldsymbol{f}\|)\|\phi\|_{\mathrm{H}^{\beta}}+|((\boldsymbol{w} \cdot \nabla) \boldsymbol{w}, \phi)| .
\end{gathered}
$$

In particular, it holds that [see (68) and (69) for further details]:

$$
\begin{aligned}
|((\boldsymbol{w} \cdot \nabla) \boldsymbol{w}, \phi)| & \leq\left|\left(\left(\boldsymbol{w}_{\mathrm{h}} \cdot \nabla_{\mathrm{h}}\right) \boldsymbol{w}, \phi\right)\right|+\left|\left(w_{3} \partial_{3} \boldsymbol{w}, \phi\right)\right| \\
& \leq c\left(\left\|\left(\boldsymbol{w}_{\mathrm{h}} \cdot \nabla_{\mathrm{h}}\right) \boldsymbol{w}\right\|_{\mathrm{H}^{-\beta}}+\left\|w_{3} \partial_{3} \boldsymbol{w}\right\|_{\mathrm{H}^{-\beta}}\right)\|\phi\|_{\mathrm{H}^{\beta}} \\
& \leq c\left(\left\|\Lambda^{\beta} \boldsymbol{w}_{\mathrm{h}}\right\|\left\|\nabla_{\mathrm{h}} \boldsymbol{w}\right\|+\left\|w_{3}\right\|_{\mathrm{H}^{1}}\left\|\Lambda^{\beta} \boldsymbol{w}\right\|\right)\|\phi\|_{\mathrm{H}^{\beta}} \\
& \leq c\left\|\nabla_{\mathrm{h}} \boldsymbol{w}\right\|\left(\left\|\Lambda^{\beta} \boldsymbol{w}_{\mathrm{h}}\right\|+\left\|\Lambda^{\beta} \boldsymbol{w}\right\|\right)\|\phi\|_{\mathrm{H}^{\beta}},
\end{aligned}
$$

and so

$$
\begin{aligned}
& \int_{0}^{T}\left\|\boldsymbol{w}_{t}+\alpha^{2} \Delta_{\mathrm{h}} \boldsymbol{w}_{t}\right\|_{\mathrm{H}^{-\beta}}^{2} d s \\
& \quad \leq c \int_{0}^{T}\left(v\left(\left\|\Lambda^{\beta} \boldsymbol{w}\right\|+\alpha^{2}\left\|\nabla_{\mathrm{h}} \Lambda^{\beta} \boldsymbol{w}\right\|\right)+\|\eta\|_{\mathcal{M}_{\mathrm{h}}^{1}}+\|\boldsymbol{f}\|\right)^{2} d s \\
& \quad+c \int_{0}^{T}\left\|\Lambda^{\beta} \boldsymbol{w}\right\|^{2} d s .
\end{aligned}
$$

Hence, it follows that $\left\|\boldsymbol{w}_{t}+\alpha^{2} \Delta_{\mathrm{h}} \boldsymbol{w}_{t}\right\|_{\mathrm{H}^{-\beta}}^{2} \in \mathrm{L}_{\text {loc }}^{2}(0,+\infty)$.

\section{Energy Equality, Continuous Dependence and Uniqueness}

We start by recalling the following result. Let $\boldsymbol{w}_{\varepsilon}, \eta_{\varepsilon}$ denote the standard regularization (convolution in time) of $w, \eta$, with $0<t_{0}<t_{1}<T$ fixed, and $0<\varepsilon<t_{0}, \varepsilon<T-t_{1}$, $\varepsilon<t_{1}-t_{0}$ (see [5,22]). For each $t \in\left[t_{0}, t_{1}\right]$, we have

$$
\boldsymbol{w}_{\varepsilon}(t)=\left(j_{\varepsilon} * \boldsymbol{w}\right)(t)=\int_{t_{0}}^{t_{1}} j_{\varepsilon}(t-\tau) \boldsymbol{w}(\tau) \mathrm{d} \tau,
$$

and

$$
\eta_{\varepsilon}(s):=\eta_{\varepsilon}^{t}(s)=\left(j_{\varepsilon} * \eta\right)^{t}(s)=\int_{t_{0}}^{t_{1}} j_{\varepsilon}(t-\tau) \eta^{\tau}(s) \mathrm{d} \tau,
$$

where the smooth function $j_{\varepsilon}$ is even, positive, supported in ] $-\varepsilon, \varepsilon$ [, and such that $\int_{-\varepsilon}^{\varepsilon} j_{\varepsilon}(s) \mathrm{d} s=1$. Under these assumptions, we have, for any $w \in \mathrm{L}^{q}\left(t_{0}, t_{1} ; X\right)$, with $1 \leq q<+\infty$ and $X$ Hilbert space, the following properties (see [22]):

i) $\boldsymbol{w}_{\varepsilon} \in \mathscr{C}^{\infty}\left(\left[t_{0}, t_{1}\right] ; X\right)$,

ii) $\lim _{\varepsilon \rightarrow 0}\left\|\boldsymbol{w}_{\varepsilon}-\boldsymbol{w}\right\|_{\mathrm{L}^{q}\left(t_{0}, t_{1} ; X\right)}=0$,

iii) $\lim _{k \rightarrow+\infty}\left\|\left(\boldsymbol{w}_{k}\right)_{\varepsilon}-\boldsymbol{w}_{\varepsilon}\right\|_{\mathrm{L}^{q}\left(t_{0}, t_{1} ; X\right)}=0$ for each subsequence $\boldsymbol{w}_{k} \in \mathrm{L}^{q}\left(t_{0}, t_{1} ; X\right)$ such that $\boldsymbol{w}_{k} \rightarrow \boldsymbol{w}$ in $\mathrm{L}^{q}\left(t_{0}, t_{1} ; X\right)$, 
and the same identical properties, in proper spaces, hold true for $\eta_{\varepsilon}$. Observe that the $\varepsilon$ regularization commutes with the filter $A_{\mathrm{h}}$ and more generally with space derivatives.

\subsection{Energy Equality}

Starting from initial data in $\left(\boldsymbol{w}_{0}, \eta_{0}\right) \in \mathcal{H}_{\mathrm{h}}^{1}$, we prove that the energy equality holds true for the model (28)-(30).

Consider a sequence $\left\{\left(\boldsymbol{w}_{k}, \boldsymbol{\eta}_{k}\right)\right\}$ of Galerkin approximating functions such that

$$
\begin{array}{lll}
\boldsymbol{w}_{k} \rightarrow \boldsymbol{w} & \text { in } & \mathrm{L}^{\infty}\left(0, T ; \mathrm{V}_{\mathrm{h}}\right) \cap \mathrm{L}^{2}\left(0, T ; V \cap \mathrm{H}_{\mathrm{h}}^{1+\beta}\right), \\
\eta_{k} \rightarrow \eta & \text { in } \quad \mathrm{L}^{2}\left(0, T ; \mathcal{M}_{\mathrm{h}}^{1}\right), \\
\eta_{k} \stackrel{*}{\rightarrow} \eta & \text { in } & \mathrm{L}^{\infty}\left(0, T ; \mathcal{M}_{\mathrm{h}}^{1}\right),
\end{array}
$$

then test (28) against $\boldsymbol{w}_{k, \varepsilon}$ in $\mathrm{L}^{2}$, and integrate on the interval $\left[t_{0}, t_{1}\right]$ in $d \tau$, to get:

$$
\begin{aligned}
& \int_{t_{0}}^{t_{1}}\left[\left(\boldsymbol{w}, \partial_{t} \boldsymbol{w}_{k, \varepsilon}\right)+\alpha^{2}\left(\nabla_{\mathrm{h}} \boldsymbol{w}, \partial_{t} \nabla_{\mathrm{h}} \boldsymbol{w}_{k, \varepsilon}\right)-v\left(\Lambda^{\beta} \boldsymbol{w}, \Lambda^{\beta} \boldsymbol{w}_{k, \varepsilon}\right)-v \alpha^{2}\left(\Lambda^{\beta} \nabla_{\mathrm{h}} \boldsymbol{w}, \Lambda^{\beta} \nabla_{\mathrm{h}} \boldsymbol{w}_{k, \varepsilon}\right)\right. \\
& \left.\quad+\left((\boldsymbol{w} \cdot \nabla) \boldsymbol{w}_{k, \varepsilon}, \boldsymbol{w}\right)\right](\tau) \mathrm{d} \tau-\int_{t_{0}}^{t_{1}} \int_{0}^{\infty} \mu(s)\left\langle\eta(s), \boldsymbol{w}_{k, \varepsilon}(\tau)\right\rangle_{\mathrm{V}_{\mathrm{h}}} \mathrm{d} s \mathrm{~d} \tau \\
& =-\int_{t_{0}}^{t_{1}}\left(\boldsymbol{f}, \boldsymbol{w}_{k, \varepsilon}\right)(s) \mathrm{d} s+\left(\boldsymbol{w}\left(t_{1}\right), A_{\mathrm{h}} \boldsymbol{w}_{k, \varepsilon}\left(t_{1}\right)\right)-\left(\boldsymbol{w}\left(t_{0}\right), A_{\mathrm{h}} \boldsymbol{w}_{k, \varepsilon}\left(t_{0}\right)\right)
\end{aligned}
$$

with $A_{\mathrm{h}}=I-\alpha^{2} \Delta_{\mathrm{h}}$.

Testing (29) against $\eta_{k, \varepsilon}$ in $\mathcal{M}_{\mathrm{h}}^{1}$, and then integrating on the interval $\left[t_{0}, t_{1}\right]$ in $d \tau$, we get (after using Fubini's theorem and an integration by parts):

$$
\begin{gathered}
-\int_{t_{0}}^{t_{1}} \int_{0}^{\infty} \mu(s)\left\langle\eta, \partial_{t} \eta_{k, \varepsilon}\right\rangle_{\mathrm{V}_{\mathrm{h}}} \mathrm{d} s \mathrm{~d} \tau+\int_{0}^{\infty} \mu(s)\left[\left\langle\eta^{t_{1}}, \eta_{k, \varepsilon}^{t_{1}}\right\rangle_{\mathrm{V}_{\mathrm{h}}}-\left\langle\eta^{t_{0}}, \eta_{k, \varepsilon}^{t_{0}}\right\rangle \mathrm{V}_{\mathrm{h}}\right] d s \\
+\int_{t_{0}}^{t_{1}} \int_{0}^{\infty} \mu(s)\left\langle\partial_{s} \eta, \eta_{k, \varepsilon}\right\rangle_{\mathrm{V}_{\mathrm{h}}} \mathrm{d} s \mathrm{~d} \tau-\int_{t_{0}}^{t_{1}} \int_{0}^{\infty} \mu(s)\left\langle\boldsymbol{w}(\tau), \eta_{k, \varepsilon}\right\rangle_{\mathrm{V}_{\mathrm{h}}} \mathrm{d} s \mathrm{~d} \tau=0
\end{gathered}
$$

that is

$$
\begin{aligned}
\int_{t_{0}}^{t_{1}} & \int_{0}^{\infty} \mu(s)\left\langle\eta, \partial_{t} \eta_{k, \varepsilon}\right\rangle_{\mathrm{V}_{\mathrm{h}}} \mathrm{d} s \mathrm{~d} \tau+\int_{t_{0}}^{t_{1}}\left\langle T \eta, \eta_{k, \varepsilon}\right\rangle_{\mathcal{M}_{\mathrm{h}}^{1}} \mathrm{~d} \tau \\
& +\int_{t_{0}}^{t_{1}} \int_{0}^{\infty} \mu(s)\left\langle\boldsymbol{w}(\tau), \eta_{k, \varepsilon}\right\rangle_{\mathrm{V}_{\mathrm{h}}} \mathrm{d} s \mathrm{~d} \tau \\
& =\int_{0}^{\infty} \mu(s)\left[\left\langle\eta^{t_{1}}, \eta_{k, \varepsilon}^{t_{1}}\right\rangle \mathrm{V}_{\mathrm{h}}-\left\langle\eta^{t_{0}}, \eta_{k, \varepsilon}^{t_{0}}\right\rangle \mathrm{V}_{\mathrm{h}}\right] \mathrm{d} s .
\end{aligned}
$$

Adding relations (54) and (55), we reach

$$
\begin{aligned}
\int_{t_{0}}^{t_{1}} & {\left[\left(\boldsymbol{w}, \partial_{t} \boldsymbol{w}_{k, \varepsilon}\right)+\alpha^{2}\left(\nabla_{\mathrm{h}} \boldsymbol{w}, \partial_{t} \nabla_{\mathrm{h}} \boldsymbol{w}_{k, \varepsilon}\right)\right.} \\
& -v\left(\Lambda^{\beta} \boldsymbol{w}, \Lambda^{\beta} \boldsymbol{w}_{k, \varepsilon}\right)-v \alpha^{2}\left(\Lambda^{\beta} \nabla_{\mathrm{h}} \boldsymbol{w}, \Lambda^{\beta} \nabla_{\mathrm{h}} \boldsymbol{w}_{k, \varepsilon}\right) \\
& \left.+\left((\boldsymbol{w} \cdot \nabla) \boldsymbol{w}_{k, \varepsilon}, \boldsymbol{w}\right)\right](\tau) \mathrm{d} \tau+\int_{t_{0}}^{t_{1}} \int_{0}^{\infty} \mu(s)\left\langle\partial_{t} \eta_{k, \varepsilon}, \eta\right\rangle_{\mathrm{V}_{\mathrm{h}}} \mathrm{d} s \mathrm{~d} \tau
\end{aligned}
$$




$$
\begin{aligned}
& +\int_{t_{0}}^{t_{1}}\left\langle T \eta_{k, \varepsilon}, \eta\right\rangle_{\mathcal{M}_{\mathrm{h}}^{1}} \mathrm{~d} \tau \\
& +\int_{t_{0}}^{t_{1}} \int_{0}^{\infty} \mu(s)\left[\left\langle\eta_{k, \varepsilon}, \boldsymbol{w}(\tau)\right\rangle_{\mathrm{V}_{\mathrm{h}}}-\left\langle\eta, \boldsymbol{w}_{k, \varepsilon}(\tau)\right\rangle_{\mathrm{V}_{\mathrm{h}}}\right] \mathrm{d} s \mathrm{~d} \tau \\
& =-\int_{t_{0}}^{t_{1}}\left(\boldsymbol{f}, \boldsymbol{w}_{k, \varepsilon}\right)(s) \mathrm{d} s+\left\langle\boldsymbol{w}\left(t_{1}\right), \boldsymbol{w}_{k, \varepsilon}\left(t_{1}\right)\right\rangle_{\mathrm{V}_{\mathrm{h}}}-\left\langle\boldsymbol{w}\left(t_{0}\right), \boldsymbol{w}_{k, \varepsilon}\left(t_{0}\right)\right\rangle_{\mathrm{V}_{\mathrm{h}}} \\
& +\int_{0}^{\infty} \mu(s)\left[\left\langle\eta^{t_{1}}, \eta_{k, \varepsilon}^{t_{1}}\right\rangle_{\mathrm{V}_{\mathrm{h}}}-\left\langle\eta^{t_{0}}, \eta_{k, \varepsilon}^{t_{0}}\right\rangle_{\mathrm{V}_{\mathrm{h}}}\right] \mathrm{d} s .
\end{aligned}
$$

Now, we use the same argument as in [9] (see also [22]): By taking $k \rightarrow+\infty$, and using the converges types (53), we get

$$
\begin{aligned}
\int_{t_{0}}^{t_{1}} & {\left[\left(\boldsymbol{w}, \partial_{t} \boldsymbol{w}_{\varepsilon}\right)+\alpha^{2}\left(\nabla_{\mathrm{h}} \boldsymbol{w}, \partial_{t} \nabla_{\mathrm{h}} \boldsymbol{w}_{\varepsilon}\right)-v\left(\Lambda^{\beta} \boldsymbol{w}, \Lambda^{\beta} \boldsymbol{w}_{\varepsilon}\right)-v \alpha^{2}\left(\Lambda^{\beta} \nabla_{\mathrm{h}} \boldsymbol{w}, \Lambda^{\beta} \nabla_{\mathrm{h}} \boldsymbol{w}_{\varepsilon}\right)\right.} \\
& \left.+\left((\boldsymbol{w} \cdot \nabla) \boldsymbol{w}_{\varepsilon}, \boldsymbol{w}\right)\right](\tau) \mathrm{d} \tau+\int_{t_{0}}^{t_{1}} \int_{0}^{\infty} \mu(s)\left\langle\partial_{t} \eta_{\varepsilon}, \eta\right\rangle_{\mathrm{V}_{\mathrm{h}}} \mathrm{d} s \mathrm{~d} \tau+\int_{t_{0}}^{t_{1}}\left\langle T \eta_{\varepsilon}, \eta\right\rangle_{\mathcal{M}_{\mathrm{h}}^{1}} \mathrm{~d} \tau \\
& +\int_{t_{0}}^{t_{1}} \int_{0}^{\infty} \mu(s)\left[\left\langle\eta_{\varepsilon}, \boldsymbol{w}(\tau)\right\rangle_{\mathrm{V}_{\mathrm{h}}}-\left\langle\nabla_{\mathrm{h}} \eta(s), \boldsymbol{w}_{\varepsilon}(\tau)\right\rangle_{\mathrm{V}_{\mathrm{h}}}\right] \mathrm{d} s \mathrm{~d} \tau \\
= & -\int_{t_{0}}^{t_{1}}\left(\boldsymbol{f}, \boldsymbol{w}_{\varepsilon}\right)(s) \mathrm{d} s+\left\langle\boldsymbol{w}\left(t_{1}\right), \boldsymbol{w}_{\varepsilon}\left(t_{1}\right)\right\rangle_{\mathrm{V}_{\mathrm{h}}}-\left\langle\boldsymbol{w}\left(t_{0}\right), \boldsymbol{w}_{\varepsilon}\left(t_{0}\right)\right\rangle_{\mathrm{V}_{\mathrm{h}}} \\
& +\int_{0}^{\infty} \mu(s)\left[\left\langle\eta^{t_{1}}, \eta_{\varepsilon}^{t_{1}}\right\rangle_{\mathrm{V}_{\mathrm{h}}}-\left\langle\nabla_{\mathrm{h}} \eta^{t_{0}}, \nabla_{\mathrm{h}} \eta_{\varepsilon}^{t_{0}}\right\rangle_{\mathrm{V}_{\mathrm{h}}}\right] \mathrm{d} s
\end{aligned}
$$

To pass to the limit as $\varepsilon \rightarrow 0$, we use the facts listed in the remark below

Remark 4.1 By using the regularity of $w$, we have

$$
\lim _{\varepsilon \rightarrow 0} \int_{t_{0}}^{t_{1}}\left((\boldsymbol{w} \cdot \nabla) \boldsymbol{w}_{\varepsilon}, \boldsymbol{w}\right) \mathrm{d} \tau=\int_{t_{0}}^{t_{1}}((\boldsymbol{w} \cdot \nabla) \boldsymbol{w}, \boldsymbol{w}) \mathrm{d} \tau=0,
$$

where the equality to zero is obtained in a standard way by approximating $\boldsymbol{w}$ through smooth functions and using the fact that $\nabla \cdot \boldsymbol{w}=0$.

Since $j_{\varepsilon}$ is supported in ] $-\varepsilon, \varepsilon$ [ and even, so that its derivative $j_{\varepsilon}^{\prime}:=\left(j_{\varepsilon}\right)^{\prime}$ is odd. Recalling the definition of $\boldsymbol{w}_{\varepsilon}$, we infer

$$
\begin{aligned}
\int_{t_{0}}^{t_{1}}\left(\boldsymbol{w}(\tau), \partial_{t} \boldsymbol{w}_{\varepsilon}(\tau)\right) \mathrm{d} \tau & =\int_{t_{0}}^{t_{1}} \int_{t_{0}}^{t_{1}} j_{\varepsilon}^{\prime}(\tau-r)(\boldsymbol{w}(\tau), \boldsymbol{w}(r)) \mathrm{d} \tau \mathrm{d} r \\
& =\iint_{E_{1}}+\iint_{E_{2}} j_{\varepsilon}^{\prime}(\tau-r)(\boldsymbol{w}(\tau), \boldsymbol{w}(r)) \mathrm{d} \tau \mathrm{d} r=0,
\end{aligned}
$$

where, in the first integral in the second line, the integrand is the same as in the second one but omitted for brevity, and

$$
\begin{aligned}
& E_{1}=\left\{(r, \tau) \in\left[t_{0}, t_{1}\right] \times\left[t_{0}, t_{1}\right]: r \leq \tau \leq r+\varepsilon\right\}, \\
& E_{2}=\left\{(r, \tau) \in\left[t_{0}, t_{1}\right] \times\left[t_{0}, t_{1}\right]: r-\varepsilon \leq \tau \leq r, r \leq t_{1}\right\} .
\end{aligned}
$$

Indeed, note that $E_{1}$ is symmetric to $E_{2}$ with respect to $\tau=r$, and $j_{\varepsilon}^{\prime}(\tau-r)$ is odd with respect to $\tau-r$, hence $\iint_{E_{2}}=-\iint_{E_{1}}$. 
Similarly, after using Fubini's theorem and the previous mentioned symmetries, we have that

$$
\begin{aligned}
\int_{t_{0}}^{t_{1}} & \int_{0}^{\infty} \mu(s)\left\langle\partial_{t} \eta_{\varepsilon}(s), \eta(s)\right\rangle_{\mathrm{V}_{\mathrm{h}}} \mathrm{d} s \mathrm{~d} \tau \\
\quad= & \int_{t_{0}}^{t_{1}} \int_{0}^{\infty} \mu(s)\left\langle\partial_{t} \eta_{\varepsilon}^{\tau}(s), \eta^{\tau}(s)\right\rangle_{\mathrm{V}_{\mathrm{h}}} \mathrm{d} s \mathrm{~d} \tau \\
& =\int_{0}^{\infty} \mu(s)\left(\int_{t_{0}}^{t_{1}} \int_{t_{0}}^{t_{1}} j_{\mathcal{\varepsilon}}^{\prime}(\tau-r)\left\langle\eta^{r}(s), \eta^{\tau}(s)\right\rangle_{\mathrm{V}_{\mathrm{h}}} \mathrm{d} \tau \mathrm{d} r\right) \mathrm{d} s \\
& =\int_{0}^{\infty} \mu(s)\left(\iint_{E_{1}}+\iint_{E_{2}} j_{\varepsilon}^{\prime}(\tau-r)\langle\eta(s), \eta(s)\rangle_{\mathrm{V}_{\mathrm{h}}} \mathrm{d} \tau \mathrm{d} r\right) \mathrm{d} s \\
& =0 .
\end{aligned}
$$

Also, we have that $\left(\nabla_{\mathrm{h}} \boldsymbol{w}\left(t_{1}\right), \nabla_{\mathrm{h}} \boldsymbol{w}_{\varepsilon}\left(t_{1}\right)\right)=\frac{1}{2}\left\|\nabla_{\mathrm{h}} \boldsymbol{w}\left(t_{1}\right)\right\|^{2}+O(\varepsilon)$ as well as $\left(\boldsymbol{w}\left(t_{0}\right), \boldsymbol{w}_{\varepsilon}\left(t_{0}\right)\right)$ $=\frac{1}{2}\left\|\boldsymbol{w}\left(t_{0}\right)\right\|^{2}+O(\varepsilon)$. Finally, it holds true that

$$
\int_{0}^{\infty} \mu(s)\left[\left\langle\eta^{t_{1}}, \eta_{\varepsilon}^{t_{1}}\right\rangle_{\mathrm{V}_{\mathrm{h}}}-\left\langle\eta^{t_{0}}, \eta_{\varepsilon}^{t_{0}}\right\rangle_{\mathrm{V}_{\mathrm{h}}}\right] \mathrm{d} s=\frac{1}{2}\left(\left\|\eta^{t_{1}}\right\|_{\mathcal{M}_{\mathrm{h}}^{1}}^{2}-\left\|\eta^{t_{0}}\right\|_{\mathcal{M}_{\mathrm{h}}^{1}}^{2}\right)+O(\varepsilon)
$$

The remaining terms in (56) can be handled in a similar way.

Then, in light of Remark 4.1, passing to the limit as $\varepsilon \rightarrow 0$ in (56), we find

$$
\begin{gathered}
\frac{1}{2}\left(\left\|\boldsymbol{w}\left(t_{1}\right)\right\|_{\mathrm{V}_{\mathrm{h}}}^{2}+\left\|\eta^{t_{1}}\right\|_{\mathcal{M}_{\mathrm{h}}^{1}}^{2}\right)+v \int_{t_{0}}^{t_{1}}\left[\left\|\Lambda^{\beta} \boldsymbol{w}\right\|^{2}+\alpha^{2}\left\|\Lambda^{\beta} \nabla_{\mathrm{h}} \boldsymbol{w}\right\|^{2}\right](\tau) \mathrm{d} \tau-\int_{t_{0}}^{t_{1}}\langle T \eta, \eta\rangle_{\mathcal{M}_{\mathrm{h}}^{1}} \mathrm{~d} \tau \\
=\int_{t_{0}}^{t_{1}}(\boldsymbol{f}, \boldsymbol{w})(\tau) \mathrm{d} \tau+\frac{1}{2}\left(\left\|\boldsymbol{w}\left(t_{0}\right)\right\|_{\mathrm{V}_{\mathrm{h}}}^{2}+\left\|\eta^{t_{0}}\right\|_{\mathcal{M}_{\mathrm{h}}^{1}}^{2}\right)
\end{gathered}
$$

which is the appropriate version of the energy equality for the considered model.

\subsection{Continuous Dependence and Uniqueness}

To study the continuous dependence on initial data, let us consider two solutions $\left(\boldsymbol{w}_{1}, \eta_{1}\right)$ and $\left(\boldsymbol{w}_{2}, \eta_{2}\right)$ to (28)-(29), and set $\boldsymbol{w}=\boldsymbol{w}_{1}-\boldsymbol{w}_{2}$ and $\eta=\eta_{1}-\eta_{2}$. Then, we test the equations for $\boldsymbol{w}$ against $\left(A_{\mathrm{h}} \boldsymbol{w}\right)_{k, \varepsilon}$ (note that $A_{\mathrm{h}} \boldsymbol{w}$ and $A_{\mathrm{h}} \eta$ are not directly allowed as test functions). Proceeding as in the previous subsection, we can pass to the limit $k \rightarrow+\infty$, to get

$$
\begin{aligned}
& \int_{0}^{t}\left\{\left(\boldsymbol{w}, \partial_{t} \boldsymbol{w}_{\varepsilon}\right)+\alpha^{2}\left(\nabla_{\mathrm{h}} \boldsymbol{w}, \partial_{t} \nabla_{\mathrm{h}} \boldsymbol{w}_{\varepsilon}\right)-v\left(\Lambda^{\beta} \boldsymbol{w}, \Lambda^{\beta} \boldsymbol{w}_{\varepsilon}\right)-v \alpha^{2}\left(\Lambda^{\beta} \nabla_{\mathrm{h}} \boldsymbol{w}, \Lambda^{\beta} \nabla_{\mathrm{h}} w_{\varepsilon}\right)\right. \\
& \left.\quad+\left(\left(\boldsymbol{w}_{1} \cdot \nabla\right) \boldsymbol{w}_{\varepsilon}, \boldsymbol{w}_{1}\right)-\left(\left(\boldsymbol{w}_{2} \cdot \nabla\right) \boldsymbol{w}_{\varepsilon}, \boldsymbol{w}_{2}\right)\right\}(s) d s-\int_{0}^{t} \int_{0}^{\infty} \mu(s)\left\langle\eta(s), \boldsymbol{w}_{\varepsilon}(\tau)\right\rangle_{\mathrm{V}_{\mathrm{h}}} \mathrm{d} s \mathrm{~d} \tau \\
& =\left(\boldsymbol{w}(t), \boldsymbol{w}_{\varepsilon}(t)\right)+\alpha^{2}\left(\nabla_{\mathrm{h}} \boldsymbol{w}(t), \nabla_{\mathrm{h}} \boldsymbol{w}_{\varepsilon}(t)\right)-\left(\boldsymbol{w}(0), \boldsymbol{w}_{\varepsilon}(0)\right)-\alpha^{2}\left(\nabla_{\mathrm{h}} \boldsymbol{w}(0), \nabla_{\mathrm{h}} \boldsymbol{w}_{\varepsilon}(0)\right)
\end{aligned}
$$


and so

$$
\begin{aligned}
& \int_{0}^{t}\left\{\left(\boldsymbol{w}, \partial_{t} \boldsymbol{w}_{\varepsilon}\right)+\alpha^{2}\left(\nabla_{\mathrm{h}} \boldsymbol{w}, \partial_{t} \nabla_{\mathrm{h}} \boldsymbol{w}_{\varepsilon}\right)-v\left(\Lambda^{\beta} \boldsymbol{w}, \Lambda^{\beta} \boldsymbol{w}_{\varepsilon}\right)-v \alpha^{2}\left(\Lambda^{\beta} \nabla_{\mathrm{h}} \boldsymbol{w}, \Lambda^{\beta} \nabla_{\mathrm{h}} w_{\varepsilon}\right)\right. \\
& \left.\quad+\left((\boldsymbol{w} \cdot \nabla) \boldsymbol{w}_{\varepsilon}, \boldsymbol{w}\right)+\left(\left(\boldsymbol{w}_{2} \cdot \nabla\right) \boldsymbol{w}_{\varepsilon}, \boldsymbol{w}\right)+\left((\boldsymbol{w} \cdot \nabla) \boldsymbol{w}_{\varepsilon}, \boldsymbol{w}_{2}\right)\right\}(s) d s \\
& \quad-\int_{0}^{t} \int_{0}^{\infty} \mu(s)\left\langle\eta(s), \boldsymbol{w}_{\varepsilon}(\tau)\right\rangle_{\mathrm{V}_{\mathrm{h}}} \mathrm{d} s \mathrm{~d} \tau \\
& =\left(\boldsymbol{w}(t), \boldsymbol{w}_{\varepsilon}(t)\right)+\alpha^{2}\left(\nabla_{\mathrm{h}} \boldsymbol{w}(t), \nabla_{\mathrm{h}} \boldsymbol{w}_{\varepsilon}(t)\right)-\left(\boldsymbol{w}(0), \boldsymbol{w}_{\varepsilon}(0)\right)-\alpha^{2}\left(\nabla_{\mathrm{h}} \boldsymbol{w}(0), \nabla_{\mathrm{h}} \boldsymbol{w}_{\varepsilon}(0)\right) .
\end{aligned}
$$

To pass to the limit as $\varepsilon \rightarrow 0$, we observe that, by proceeding as in (57) and following, we have that

$$
\begin{aligned}
& \text { i) } \int_{0}^{t}\left\{\left(\boldsymbol{w}, \partial_{t} \boldsymbol{w}_{\varepsilon}\right)+\alpha^{2}\left(\nabla_{\mathrm{h}} \boldsymbol{w}, \partial_{t} \nabla_{\mathrm{h}} \boldsymbol{w}_{\varepsilon}\right)\right\}(s) d s=0, \\
& \text { ii) } \lim _{\varepsilon \rightarrow 0} \int_{0}^{t}\left\{v\left(\Lambda^{\beta} \boldsymbol{w}, \Lambda^{\beta} \boldsymbol{w}_{\varepsilon}\right)+v \alpha^{2}\left(\Lambda^{\beta} \nabla_{\mathrm{h}} \boldsymbol{w}, \Lambda^{\beta} \nabla_{\mathrm{h}} w_{\varepsilon}\right)\right\}(s) d s \\
& =v \int_{0}^{t}\left\{\left\|\Lambda^{\beta} \boldsymbol{w}\right\|^{2}+\alpha^{2}\left\|\Lambda^{\beta} \nabla_{\mathrm{h}} \boldsymbol{w}\right\|\right\}(s) d s, \\
& \text { iii) } \left.\lim _{\varepsilon \rightarrow 0} \int_{0}^{t}\left((\boldsymbol{w} \cdot \nabla) \boldsymbol{w}_{\varepsilon}, \boldsymbol{w}\right)+\left(\left(\boldsymbol{w}_{2} \cdot \nabla\right) \boldsymbol{w}_{\varepsilon}, \boldsymbol{w}\right)+\left((\boldsymbol{w} \cdot \nabla) \boldsymbol{w}_{\varepsilon}, \boldsymbol{w}_{2}\right)\right\}(s) d s \\
& =\int_{0}^{t}\left((\boldsymbol{w} \cdot \nabla) \boldsymbol{w}, \boldsymbol{w}_{2}\right)(s) d s, \\
& \text { iv) } \lim _{\varepsilon \rightarrow 0} \int_{0}^{t} \int_{0}^{\infty} \mu(s)\left\langle\eta(s), \boldsymbol{w}_{\varepsilon}(\tau)\right\rangle_{\mathrm{V}_{\mathrm{h}}} \mathrm{d} s \mathrm{~d} \tau \\
& =\int_{0}^{t} \int_{0}^{\infty} \mu(s)\langle\eta(s), w(\tau)\rangle_{\mathrm{V}_{\mathrm{h}}} \mathrm{d} s \mathrm{~d} \tau
\end{aligned}
$$

and, in particular, we have that

$$
\begin{gathered}
\left(\boldsymbol{w}(t), \boldsymbol{w}_{\varepsilon}(t)\right)+\alpha^{2}\left(\nabla_{\mathrm{h}} \boldsymbol{w}(t), \nabla_{\mathrm{h}} \boldsymbol{w}_{\varepsilon}(t)\right)-\left(\boldsymbol{w}(0), \boldsymbol{w}_{\varepsilon}(0)\right)-\alpha^{2}\left(\nabla_{\mathrm{h}} \boldsymbol{w}(0), \nabla_{\mathrm{h}} \boldsymbol{w}_{\varepsilon}(0)\right) \\
=\frac{1}{2}\left(\|\boldsymbol{w}(t)\|^{2}+\alpha^{2}\left\|\nabla_{\mathrm{h}} \boldsymbol{w}(t)\right\|^{2}-\|\boldsymbol{w}(0)\|^{2}-\alpha^{2}\left\|\nabla_{\mathrm{h}} \boldsymbol{w}(0)\right\|^{2}\right)+O(\varepsilon) .
\end{gathered}
$$

Hence, using (59) along with the above relations, we obtain

$$
\begin{aligned}
& \frac{1}{2}\left(\|\boldsymbol{w}(t)\|^{2}+\alpha^{2}\left\|\nabla_{\mathrm{h}} \boldsymbol{w}(t)\right\|^{2}\right)+v \int_{0}^{t}\left(\left\|\Lambda^{\beta} \boldsymbol{w}\right\|^{2}+\alpha^{2}\left\|\Lambda^{\beta} \nabla_{\mathrm{h}} \boldsymbol{w}\right\|\right)(s) d s \\
& \quad+\int_{0}^{t} \int_{0}^{\infty} \mu(s)\langle\eta(s), \boldsymbol{w}(\tau)\rangle_{\mathrm{V}_{\mathrm{h}}} \mathrm{d} s \mathrm{~d} \tau \\
& \leq\left|\int_{0}^{t}\left((\boldsymbol{w} \cdot \nabla) \boldsymbol{w}, \boldsymbol{w}_{2}\right)(s) d s\right|+\frac{1}{2}\left(\|\boldsymbol{w}(0)\|^{2}+\alpha^{2}\left\|\nabla_{\mathrm{h}} \boldsymbol{w}(0)\right\|^{2}\right) .
\end{aligned}
$$

Now, using the fact that $\eta$ satisfies the representation formula (32) (see $[16,19]$ ) it follows that

$$
\frac{1}{2}\left\|\eta^{t}\right\|_{\mathcal{M}_{\mathrm{h}}^{1}}^{2}-\frac{1}{2}\left\|\eta^{0}\right\|_{\mathcal{M}_{\mathrm{h}}^{1}}^{2} \leq \int_{0}^{t}\left\langle\eta^{\tau}, \boldsymbol{w}(\tau)\right\rangle_{\mathcal{M}_{\mathrm{h}}^{1}} d \tau .
$$

Also, the nonlinear term in the right-hand side of (60) can be controlled as follows: 


$$
\begin{aligned}
\left|\left((\boldsymbol{w} \cdot \nabla) \boldsymbol{w}, \boldsymbol{w}_{2}\right)\right| & \leq C\left(\left\|\boldsymbol{w}_{\mathrm{h}} \cdot \nabla_{\mathrm{h}} \boldsymbol{w}\right\|_{\mathrm{H}^{-\beta}}+\left\|w_{3} \partial_{3} \boldsymbol{w}\right\|_{\mathrm{H}^{-\beta}}\right)\left\|\boldsymbol{w}_{2}\right\|_{\mathrm{H}^{\beta}} \\
& \leq C\left(\left\|\boldsymbol{w}_{\mathrm{h}}\right\|_{\mathrm{H}^{\beta}}\left\|\nabla_{\mathrm{h}} \boldsymbol{w}\right\|+\left\|w_{3}\right\|_{\mathrm{H}^{1}}\left\|\partial_{3} \boldsymbol{w}\right\|_{\mathrm{H}^{\beta-1}}\right)\left\|\boldsymbol{w}_{2}\right\|_{\mathrm{H}^{\beta}} \\
& \leq C\left(\left\|\Lambda^{\beta} \boldsymbol{w}\right\|\left\|\nabla_{\mathrm{h}} \boldsymbol{w}\right\|+\left\|\nabla_{\mathrm{h}} \boldsymbol{w}\right\|\left\|\Lambda^{\beta} \boldsymbol{w}\right\|\right)\left\|\boldsymbol{w}_{2}\right\|_{\mathrm{H}^{\beta}} \\
& \leq C\left\|\nabla_{\mathrm{h}} \boldsymbol{w}\right\|^{2}\left\|\boldsymbol{w}_{2}\right\|_{\mathrm{H}^{\beta}}^{2}+\frac{v}{2}\left\|\Lambda^{\beta} \boldsymbol{w}\right\|^{2}
\end{aligned}
$$

where we have used duality, Lemma 2.2 with $3 / 4 \leq \beta<1, \partial_{3} w_{3}=-\nabla_{\mathrm{h}} \boldsymbol{w}_{\mathrm{h}}$ (so that $\left.\left\|w_{3}\right\|_{\mathrm{H}^{1}} \leq C\left\|\nabla_{\mathrm{h}} \boldsymbol{w}\right\|\right)$, and the Young inequality. Hence

$$
\begin{aligned}
\int_{0}^{t}\left|\left((\boldsymbol{w} \cdot \nabla) \boldsymbol{w}, \boldsymbol{w}_{2}\right)(s)\right| d s \leq & \frac{v}{2} \int_{0}^{t}\left(\left\|\Lambda^{\beta} \boldsymbol{w}(s)\right\|^{2}+\alpha^{2}\left\|\Lambda^{\beta} \nabla_{\mathrm{h}} \boldsymbol{w}(s)\right\|^{2}\right) d s \\
& +C \int_{0}^{t}\left\|\boldsymbol{w}_{2}\right\|_{\mathrm{H}^{\beta}}^{2}\left\|\nabla_{\mathrm{h}} \boldsymbol{w}\right\|^{2} d s .
\end{aligned}
$$

Then, using (60) together with (61) and (62), we reach

$$
\begin{aligned}
& \frac{1}{2}\left(\|\boldsymbol{w}(t)\|^{2}+\alpha^{2}\left\|\nabla_{\mathrm{h}} \boldsymbol{w}(t)\right\|^{2}+\left\|\eta^{t}\right\|_{\mathcal{M}_{\mathrm{h}}^{1}}^{2}\right)+\frac{v}{2} \int_{0}^{t}\left(\left\|\Lambda^{\beta} \boldsymbol{w}\right\|^{2}+\alpha^{2}\left\|\Lambda^{\beta} \nabla_{\mathrm{h}} \boldsymbol{w}\right\|\right)(s) d s \\
& \leq C \int_{0}^{t}\left\|\boldsymbol{w}_{2}\right\|_{\mathrm{H}^{\beta}}^{2}\left(\|\boldsymbol{w}\|^{2}+\alpha^{2}\left\|\nabla_{\mathrm{h}} \boldsymbol{w}\right\|^{2}+\left\|\eta^{s}\right\|_{\mathcal{M}_{\mathrm{h}}^{1}}^{2}\right) d s \\
& \quad+\frac{1}{2}\left(\|\boldsymbol{w}(0)\|^{2}+\alpha^{2}\left\|\nabla_{\mathrm{h}} \boldsymbol{w}(0)\right\|^{2}+\frac{1}{2}\left\|\eta^{0}\right\|_{\mathcal{M}_{\mathrm{h}}^{1}}^{2}\right),
\end{aligned}
$$

and the conclusion follows by an application of Gronwall's lemma.

Remark 4.2 Thanks to an argument very close to the one just used for the continuous dependence, we can also conclude about uniqueness (see [9] for details).

Considering more regular initial data, for instance $\left(\boldsymbol{w}_{0}, \eta_{0}\right) \in \mathcal{H}_{\mathrm{h}}^{1+\beta}$ or $\left(\boldsymbol{w}_{0}, \eta_{0}\right) \in \mathcal{H}_{\mathrm{h}}^{1+2 \beta}$, we can reproduce the previous scheme - with improved properties for the considered solutions - still proving continuous dependence on initial data. When we take into account the case of $\left(\boldsymbol{w}_{0}, \eta_{0}\right) \in \mathcal{H}_{0}^{1+\beta}$, we consider again two solutions $\left(\boldsymbol{w}_{1}, \eta_{1}\right)$ and $\left(\boldsymbol{w}_{2}, \eta_{2}\right)$ to (28)-(29), with $\boldsymbol{w}=\boldsymbol{w}_{1}-\boldsymbol{w}_{2}$ and $\eta=\eta_{1}-\eta_{2}$, and we test the equation for $\boldsymbol{w}$ against $\left(A_{\mathrm{h}} \Lambda^{2 \beta} \boldsymbol{w}\right)_{k, \varepsilon}$. The argument follows by performing the same calculations previously presented in this subsection, where the only significant difference is the estimate for the nonlinear term $((\boldsymbol{w}$. $\left.\nabla) \boldsymbol{w}, \Lambda^{2 \beta} \boldsymbol{w}\right)$. The way to handle this term (and even higher order versions) is shown in Sect. 5; see (68), (86) and the subsequent computations for the details.

\section{Dissipation and Absorbing Sets}

We now proceed as in [11] (see also [15,30,31])

Lemma 5.1 There exists a bounded absorbing set $\mathcal{B}_{0} \subseteq \mathcal{H}_{\mathrm{h}}^{1}$ of radius $R_{0}=\sqrt{2 \hat{c}}\left\|\Lambda^{-\beta} \boldsymbol{f}\right\|$. In particular,

$$
\mathcal{B}_{0}=\left\{U=(\boldsymbol{w}, \eta) \in \mathcal{H}_{\mathrm{h}}^{1}: \int_{0}^{1}\|U(s)\|_{\mathcal{H}_{\mathrm{h}}^{1}}^{2} d s \leq 2 \hat{c}\left\|\Lambda^{-\beta} \boldsymbol{f}\right\|^{2}\right\},
$$

where $\hat{c}>0$ is a suitable constant. 
Proof Let $B_{0}$ be another bounded set in $\mathcal{H}_{\mathrm{h}}^{1}$. There exists $L_{0}$ such that $\int_{0}^{t}\|U(s)\|_{\mathcal{H}_{\mathrm{h}}^{1}}^{2} d s \leq L_{0}$, for all $U \in B_{0}$. Take $U \in B_{0}, t_{0} \in[0,1], t>0$, then thanks to (50) we have that

$$
\left\|S(t) U\left(t_{0}\right)\right\|_{\mathcal{H}_{\mathrm{h}}^{1}}^{2}=\left\|U\left(t+t_{0}\right)\right\|_{\mathcal{H}_{\mathrm{h}}^{1}}^{2} \leq \mathcal{Q}\left(\left\|U\left(t_{0}\right)\right\|_{\mathcal{H}_{\mathrm{h}}^{1}}^{2}\right) e^{-\hat{v} t}+c\left\|\Lambda^{-\beta} \boldsymbol{f}\right\|^{2},
$$

Now integrating in $t_{0}$ over $(0,1)$, we obtain that (up to take a larger constant than $L_{0}$, we also have that $\int_{0}^{t} \mathcal{Q}\left(\|U(s)\|_{\mathcal{H}_{\mathrm{h}}^{1}}^{2}\right) d s \leq L_{0}$, for all $\left.U \in B_{0}\right)$ the following relation holds true:

$$
\int_{0}^{1}\left\|S(t) U\left(t_{0}\right)\right\|_{\mathcal{H}_{\mathrm{h}}^{1}}^{2} d t_{0} \leq L_{0} e^{-\hat{v} t}+\hat{c}\left\|\Lambda^{-\beta} \boldsymbol{f}\right\|^{2} .
$$

We can take $t_{0}$ such that

$$
L_{0} e^{-\hat{v} t_{0}} \leq \hat{c}\left\|\Lambda^{-\beta} \boldsymbol{f}\right\|^{2}, \text { and so } t_{0} \geq \hat{v}^{-1} \ln \left(\frac{L_{0}}{\hat{c}\left\|\Lambda^{-\beta} \boldsymbol{f}\right\|^{2}}\right) .
$$

As a consequence $S(t)(U) \in \mathcal{B}_{0}$, for any $t \geq t_{0}$.

\subsection{Dissipation in $\mathcal{H}_{\mathrm{h}}^{1+\beta}$}

Here we prove the existence of an absorbing ball in $\mathcal{H}_{\mathrm{h}}^{1+\beta}=\mathrm{H}_{\mathrm{h}}^{1+\beta} \times \mathcal{M}_{\mathrm{h}}^{1+\beta}$ for the system (28)-(30). Assume $\left(\boldsymbol{w}_{0}, \eta_{0}\right) \in \mathcal{H}_{\mathrm{h}}^{1+\beta}$.

Theorem 5.2 Let $\beta \geq 3 / 4$, and let $f \in \mathrm{L}^{2}\left(0,+\infty ; \mathrm{L}^{2}\right)$. Then, for any initial datum $U_{0}=$ $\left(\boldsymbol{w}_{0}, \eta^{0}\right) \in \mathcal{H}_{\mathrm{h}}^{1+\beta}$, the corresponding energy

$$
\mathcal{E}_{1+\beta}(t)=\frac{1}{2}\left\|S(t) U_{0}\right\|_{\mathcal{H}_{\mathrm{h}}^{1+\beta}}^{2},
$$

satisfies the estimate

$$
\mathcal{E}_{1+\beta}(t) \leq C \mathcal{Q}\left(R^{2}\right) e^{-2 \bar{v} t}+C, \quad \forall t \geq 0,
$$

where $\bar{v}$ is a constant depending only on the parameters involved in the system and on the domain, and $C=C\left(\|\boldsymbol{f}\|_{\mathrm{L}^{2}\left(\mathrm{~L}^{2}\right)},\left\|U_{0}\right\|_{\mathcal{H}_{\mathrm{h}}^{1+\beta}}\right)$.

To prove this result we first introduce some preliminary calculations and lemmas.

Testing (28) against $\Lambda^{2 \beta} \boldsymbol{w}$ in $\mathrm{L}^{2}$ and (29) against $\Lambda^{2 \beta} \eta$ in $\mathcal{M}_{\mathrm{h}}^{1}$, and proceeding as before, we reach

$$
\begin{aligned}
\frac{1}{2} & \frac{d}{d t}\left(\left\|\Lambda^{\beta} \boldsymbol{w}\right\|^{2}+\alpha^{2}\left\|\nabla_{\mathrm{h}} \Lambda^{\beta} \boldsymbol{w}\right\|^{2}+\left\|\Lambda^{\beta} \eta\right\|_{\mathcal{M}_{\mathrm{h}}^{1}}^{2}\right)+(v-\varepsilon)\left(\left\|\Lambda^{2 \beta} \boldsymbol{w}\right\|^{2}+\alpha^{2}\left\|\nabla_{\mathrm{h}} \Lambda^{2 \beta} \boldsymbol{w}\right\|^{2}\right) \\
& -\left\langle T \Lambda^{\beta} \eta, \Lambda^{\beta} \eta\right\rangle_{\mathcal{M}_{\mathrm{h}}^{1}} \\
& \leq c_{\varepsilon}\|\boldsymbol{f}\|^{2}+\left|\left((\boldsymbol{w} \cdot \nabla) \boldsymbol{w}, \Lambda^{2 \beta} \boldsymbol{w}\right)\right| .
\end{aligned}
$$

Now, observe that, up to lower order terms,

$$
\begin{aligned}
\left|\left((\boldsymbol{w} \cdot \nabla) \boldsymbol{w}, \Lambda^{2 \beta} \boldsymbol{w}\right)\right| & \leq\left(\left\|\left(\boldsymbol{w}_{\mathrm{h}} \cdot \nabla_{\mathrm{h}}\right) \boldsymbol{w}\right\|+\left\|w_{3} \partial_{3} \boldsymbol{w}\right\|\right)\left\|\Lambda^{2 \beta} \boldsymbol{w}\right\| \\
& \leq c_{\varepsilon}\left(\left\|\left(\boldsymbol{w}_{\mathrm{h}} \cdot \nabla_{\mathrm{h}}\right) \boldsymbol{w}\right\|^{2}+\left\|w_{3} \partial_{3} \boldsymbol{w}\right\|^{2}\right)+\varepsilon\left\|\Lambda^{2 \beta} \boldsymbol{w}\right\|^{2} \\
& \leq c\left\|\boldsymbol{w}_{\mathrm{h}}\right\|_{\mathrm{L}^{4}}^{2}\left\|\nabla_{\mathrm{h}} \boldsymbol{w}\right\|_{\mathrm{L}^{4}}^{2}+c\left\|w_{3}\right\|_{\mathrm{H}^{1+\beta}}^{2}\left\|\partial_{3} \boldsymbol{w}\right\|_{\mathrm{H}^{\beta-1}}^{2}+\varepsilon\left\|\Lambda^{2 \beta} \boldsymbol{w}\right\|^{2} \\
& \leq c\left\|\Lambda^{\beta} \boldsymbol{w}_{\mathrm{h}}\right\|^{2}\left\|\nabla_{\mathrm{h}} \Lambda^{\beta} \boldsymbol{w}\right\|^{2}+c\left\|w_{3}\right\|_{\mathrm{H}^{1+\beta}}^{2}\left\|\partial_{3} \boldsymbol{w}\right\|_{\mathrm{H}^{\beta-1}}^{2}+\varepsilon\left\|\Lambda^{2 \beta} \boldsymbol{w}\right\|^{2}
\end{aligned}
$$


where we used Lemma 2.2 with $s_{0}=0, s_{1}=1+\beta$, and $s_{2}=\beta-1$ and so, as a consequence of (18), we have that $0+1+\beta+\beta-1 \geq 3 / 2$ if and only if $\beta \geq 3 / 4$.

In particular, we have that

$$
\begin{aligned}
\left\|w_{3}\right\|_{\mathrm{H}^{1+\beta}}^{2}\left\|\partial_{3} \boldsymbol{w}\right\|_{\mathrm{H}^{\beta-1}}^{2} & \leq c\left\|\Lambda^{\beta} w_{3}\right\|_{\mathrm{H}^{1}}^{2}\left\|\partial_{3} \boldsymbol{w}\right\|_{\mathrm{H}^{\beta-1}}^{2} \\
& \leq c\left\|\Lambda^{\beta} w_{3}\right\|_{\mathrm{H}^{1}}^{2}\|\nabla \boldsymbol{w}\|_{\mathrm{H}^{\beta-1}}^{2} \\
& \leq c\left\|\Lambda^{\beta} \nabla w_{3}\right\|^{2}\left\|\Lambda^{\beta} \boldsymbol{w}\right\|^{2} \\
& \leq c\left\|\nabla_{\mathrm{h}} \Lambda^{\beta} \boldsymbol{w}\right\|^{2}\left\|\Lambda^{\beta} \boldsymbol{w}\right\|^{2} .
\end{aligned}
$$

Therefore, using (67) along with(68) and (69), we find

$$
\begin{aligned}
& \frac{1}{2} \frac{d}{d t}\left(\left\|\Lambda^{\beta} \boldsymbol{w}\right\|^{2}+\alpha^{2}\left\|\nabla_{\mathrm{h}} \Lambda^{\beta} \boldsymbol{w}\right\|^{2}+\left\|\Lambda^{\beta} \eta\right\|_{\mathcal{M}_{\mathrm{h}}^{1}}^{2}\right) \\
& \quad+(v-2 \varepsilon)\left(\left\|\Lambda^{2 \beta} \boldsymbol{w}\right\|^{2}+\alpha^{2}\left\|\nabla_{\mathrm{h}} \Lambda^{2 \beta} \boldsymbol{w}\right\|^{2}\right)+\frac{\delta}{2}\left\|\Lambda^{\beta} \eta\right\|_{\mathcal{M}_{\mathrm{h}}^{1}}^{2} \\
& \quad \leq c_{\varepsilon}\|\boldsymbol{f}\|^{2}+c_{\varepsilon}\left\|\Lambda^{\beta} \boldsymbol{w}\right\|^{2}\left\|\nabla_{\mathrm{h}} \Lambda^{\beta} \boldsymbol{w}\right\|^{2},
\end{aligned}
$$

and using Gronwall's inequality we obtain

$$
\begin{aligned}
& \left(\left\|\Lambda^{\beta} \boldsymbol{w}\right\|^{2}+\alpha^{2}\left\|\nabla_{\mathrm{h}} \Lambda^{\beta} \boldsymbol{w}\right\|^{2}+\left\|\Lambda^{\beta} \eta\right\|_{\mathcal{M}_{\mathrm{h}}^{1}}^{2}\right)(t) \\
& \quad+\hat{v} \int_{0}^{t}\left(\left\|\Lambda^{2 \beta} \boldsymbol{w}\right\|^{2}+\alpha^{2}\left\|\nabla_{\mathrm{h}} \Lambda^{2 \beta} \boldsymbol{w}\right\|^{2}+\left\|\Lambda^{\beta} \eta\right\|_{\mathcal{M}_{\mathrm{h}}^{1}}^{2}\right)(s) d s \\
& \quad \leq\left(\left\|\Lambda^{\beta} \boldsymbol{w}_{0}\right\|^{2}+\alpha^{2}\left\|\nabla_{\mathrm{h}} \Lambda^{\beta} \boldsymbol{w}_{0}\right\|^{2}+\left\|\Lambda^{\beta} \eta_{0}\right\|_{\mathcal{M}_{\mathrm{h}}^{1}}^{2}+c\|\boldsymbol{f}\|^{2}\right) e^{\int_{0}^{t} \mathbf{b}(s) d s},
\end{aligned}
$$

with $\mathbf{b}=\left(\|\boldsymbol{w}\|^{2}+\left\|\Lambda^{\beta} \boldsymbol{w}\right\|^{2}\right) \in \mathrm{L}^{2}(0, T)$, for any $T>0$. Hence $\boldsymbol{w} \in \mathrm{L}^{2}\left(0, T ; \mathrm{H}_{\mathrm{h}}^{1+2 \beta}\right) \cap$ $\mathrm{L}^{\infty}\left(0, T ; \mathrm{H}_{\mathrm{h}}^{1+\beta}\right), w_{3} \in \mathrm{L}^{2}\left(0, T ; \mathrm{H}^{1+2 \beta}\right) \cap \mathrm{L}^{\infty}\left(0, T ; \mathrm{H}^{1+\beta}\right)$ and $\eta \in \mathrm{L}^{\infty}\left(0, T ; \mathcal{M}_{\mathrm{h}}^{1+\beta}\right)$, for any $T>0$.

Moreover, if we assume $f \in \mathrm{L}^{2}\left(0,+\infty\right.$; $\left.\mathrm{L}^{2}\right)$, then adapting (71) accordingly, we also gain that $\Lambda^{2 \beta} w \in \mathrm{L}^{2}\left(0,+\infty ; \mathrm{V}_{\mathrm{h}}\right), \Lambda^{2 \beta} w_{3} \in \mathrm{L}^{2}\left(0,+\infty ; \mathrm{H}^{1}\right)$ : As a matter of fact, it holds true that

$$
\begin{aligned}
& \left(\left\|\Lambda^{\beta} \boldsymbol{w}\right\|^{2}+\alpha^{2}\left\|\nabla_{\mathrm{h}} \Lambda^{\beta} \boldsymbol{w}\right\|^{2}+\left\|\Lambda^{\beta} \eta\right\|_{\mathcal{M}_{\mathrm{h}}^{1}}^{2}\right)(t) \\
& \quad+\hat{v} \int_{0}^{t}\left(\left\|\Lambda^{2 \beta} \boldsymbol{w}\right\|^{2}+\alpha^{2}\left\|\nabla_{\mathrm{h}} \Lambda^{2 \beta} \boldsymbol{w}\right\|^{2}+\left\|\Lambda^{\beta} \eta\right\|_{\mathcal{M}_{\mathrm{h}}^{1}}^{2}\right)(s) d s \\
& \quad \leq\left(\left\|\Lambda^{\beta} \boldsymbol{w}_{0}\right\|^{2}+\alpha^{2}\left\|\nabla_{\mathrm{h}} \Lambda^{\beta} \boldsymbol{w}_{0}\right\|^{2}+\left\|\Lambda^{\beta} \eta_{0}\right\|_{\mathcal{M}_{\mathrm{h}}^{1}}^{2}+c \int_{0}^{\infty}\|\boldsymbol{f}\|^{2} d t\right) e^{\int_{0}^{t} \mathbf{b}(s) d s} \leq C .
\end{aligned}
$$

Lemma 5.3 Assume $f \in \mathrm{L}^{2}\left(0,+\infty, \mathrm{L}^{2}\right)$. There exists $R_{1}>0$ such that, for any given $R \geq 0$, there is a nonnegative function $\psi$ vanishing at infinity such that

$$
\left\|S(t) U_{0}\right\|_{\mathcal{H}_{\mathrm{h}}^{1+\beta}} \leq \psi(t)+R_{1}, \quad \forall U_{0}=\left(\boldsymbol{w}_{0}, \eta_{0}\right) \in \mathrm{B}_{\mathcal{H}_{\mathrm{h}}^{1+\beta}}(R) .
$$

Here $\psi(t)=C \mathcal{Q}\left(R^{2}\right) e^{-2 \bar{v} t}$, with $\bar{v}=\bar{v}(v, \delta)$ and $C=C\left(\|\boldsymbol{f}\|_{\mathrm{L}^{2}\left(\mathrm{~L}^{2}\right)},\left\|U_{0}\right\|_{\mathcal{H}_{\mathrm{h}}^{1+\beta}}\right)$.

Proof Given $R \geq 0$, let us consider the ball $\mathrm{B}_{\mathcal{H}_{\mathrm{h}}^{1+\beta}}(R)$. We easily infer from the continuous embedding $\mathcal{H}_{\mathrm{h}}^{1+\beta} \subset \mathcal{H}_{\mathrm{h}}^{1}$ that $\mathrm{B}_{\mathcal{H}_{\mathrm{h}}^{1+\beta}}(R) \subset \mathrm{B}_{\mathcal{H}_{\mathrm{h}}^{1}}(\mathcal{Q}(R))$. Therefore, on account of (50), 
there exists $t_{e}=t_{e}(R)$ such that

$$
\left\{\begin{array}{l}
\left\|S(t) \mathrm{B}_{\mathcal{H}_{\mathrm{h}}^{1+\beta}}(R)\right\| \leq \mathcal{Q}(R), \quad \forall t \leq t_{e}, \\
\left\|S(t) \mathrm{B}_{\mathcal{H}_{\mathrm{h}}^{1+\beta}}(R)\right\| \leq R_{0}, \quad \forall t \geq t_{e} .
\end{array}\right.
$$

Taking an arbitrary $U_{0} \in \mathrm{B}_{\mathcal{H}_{\mathrm{h}}^{1+\beta}}(R)$, we consider the higher-order energy functional introduced in (65), i.e.

$$
\mathcal{E}_{1+\beta}(t)=\frac{1}{2}\left\|S(t) U_{0}\right\|_{\mathcal{H}_{\mathrm{h}}^{1+\beta}}^{2} .
$$

Now, using (71) along with (74), for $t \leq t_{e}$, we get

$$
\begin{aligned}
& \left(\left\|\Lambda^{\beta} \boldsymbol{w}\right\|^{2}+\alpha^{2}\left\|\nabla_{\mathrm{h}} \Lambda^{\beta} \boldsymbol{w}\right\|^{2}+\left\|\Lambda^{\beta} \eta\right\|_{\mathcal{M}_{\mathrm{h}}^{1}}^{2}\right)(t) \\
& \quad \leq\left(\left\|\Lambda^{\beta} \boldsymbol{w}_{0}\right\|^{2}+\alpha^{2}\left\|\nabla_{\mathrm{h}} \Lambda^{\beta} \boldsymbol{w}_{0}\right\|^{2}+\left\|\Lambda^{\beta} \eta_{0}\right\|_{\mathcal{M}_{\mathrm{h}}^{1}}^{2}+c\|\boldsymbol{f}\|^{2}\right) e^{\int_{0}^{t} \mathbf{b}(s) d s}, \\
& \quad \leq \mathcal{Q}\left(R^{2}\right) e^{\int_{0}^{t} \mathbf{b}(s) d s}
\end{aligned}
$$

and combining with (49), we obtain the following relation, for any $t \leq t_{e}$, i.e.

$$
\mathcal{E}_{1+\beta}(t) \leq C \mathcal{Q}\left(R^{2}\right) \mathrm{e}^{\mathcal{Q}\left(\left(R+t_{e}\right)^{2}\right)}=: C \mathcal{Q}\left(R^{2}\right), \quad \forall t \leq t_{e}
$$

Let us consider the case of $t \geq t_{e}$. By using (70) along with (43), we find

$$
\begin{aligned}
& \frac{1}{2} \frac{d}{d t}\left(\left\|\Lambda^{\beta} \boldsymbol{w}\right\|^{2}+\alpha^{2}\left\|\nabla_{\mathrm{h}} \Lambda^{\beta} \boldsymbol{w}\right\|^{2}+\left\|\Lambda^{\beta} \eta\right\|_{\mathcal{M}_{\mathrm{h}}^{1}}^{2}\right) \\
& \quad+\bar{v}\left(\left\|\Lambda^{\beta} \boldsymbol{w}\right\|^{2}+\alpha^{2}\left\|\nabla_{\mathrm{h}} \Lambda^{\beta} \boldsymbol{w}\right\|^{2}+\left\|\Lambda^{\beta} \eta\right\|_{\mathcal{M}_{\mathrm{h}}^{1}}^{2}\right) \\
& \leq c_{\varepsilon}\|\boldsymbol{f}\|^{2}+c_{\varepsilon}\left(\|\boldsymbol{w}\|^{2}+\left\|\Lambda^{\beta} \boldsymbol{w}\right\|^{2}\right)\left\|\nabla_{\mathrm{h}} \Lambda^{\beta} \boldsymbol{w}\right\|^{2}
\end{aligned}
$$

with $\bar{v}=\min \{(v-3 \varepsilon), \delta / 2\}$. Then, assuming $f \in \mathrm{L}^{2}\left(0,+\infty, \mathrm{L}^{2}\right)$, and exploiting relation (72) we have that:

$$
\begin{aligned}
& \frac{d}{d t}\left(\left\|\Lambda^{\beta} \boldsymbol{w}\right\|^{2}+\alpha^{2}\left\|\nabla_{\mathrm{h}} \Lambda^{\beta} \boldsymbol{w}\right\|^{2}+\left\|\Lambda^{\beta} \eta\right\|_{\mathcal{M}_{\mathrm{h}}^{1}}^{2}\right) \\
& \quad+2 \bar{v}\left(\left\|\Lambda^{\beta} \boldsymbol{w}\right\|^{2}+\alpha^{2}\left\|\nabla_{\mathrm{h}} \Lambda^{\beta} \boldsymbol{w}\right\|^{2}+\left\|\Lambda^{\beta} \eta\right\|_{\mathcal{M}_{\mathrm{h}}^{1}}^{2}\right) \\
& \leq c_{\varepsilon}\|\boldsymbol{f}\|^{2}+C
\end{aligned}
$$

and so

$$
\begin{aligned}
\left\|\Lambda^{\beta} \boldsymbol{w}\right\|^{2}+\alpha^{2}\left\|\nabla_{\mathrm{h}} \Lambda^{\beta} \boldsymbol{w}\right\|^{2}+\left\|\Lambda^{\beta} \eta\right\|_{\mathcal{M}_{\mathrm{h}}^{1}}^{2} & \leq C \mathcal{Q}\left(R^{2}\right) e^{-2 \bar{v}\left(t-t_{e}\right)}+\int_{0}^{\infty}\|\boldsymbol{f}\|^{2}+C \\
& \leq C \mathcal{Q}\left(R^{2}\right) e^{-2 \bar{v}\left(t-t_{e}\right)}+C, \quad \forall t \geq t_{e},
\end{aligned}
$$

where $\mathcal{Q}\left(R^{2}\right)$ is as in (76). Hence

$$
\mathcal{E}_{1+\beta}(t) \leq C \mathcal{Q}\left(R^{2}\right) e^{-2 \bar{v}\left(t-t_{e}\right)}+C, \quad \forall t \geq t_{e},
$$

and using together (76) and (80), we finally get

$$
\mathcal{E}_{1+\beta}(t) \leq C \mathcal{Q}\left(R^{2}\right) e^{-2 \bar{v} t}+C, \quad \forall t \geq 0 .
$$

This proves Lemma 5.3, after setting $\psi(t)=C \mathcal{Q}\left(R^{2}\right) e^{-2 \bar{v} t}$ and $R_{1}=C=$ $C\left(\|\boldsymbol{f}\|_{\mathrm{L}^{2}\left(\mathrm{~L}^{2}\right)},\left\|U_{0}\right\|_{\mathcal{H}_{\mathrm{h}}^{1+\beta}}\right)$. 
The Proof of Theorem 5.2 follows by Lemma 5.3.

In the next section, we will use the following results concerning the existence of a higher order absorbing ball and a higher order uniform control. Using the same argument as in Lemma 5.1, we can prove that

Lemma 5.4 There exists a bounded absorbing set $\overline{\mathcal{B}}_{0} \subseteq \mathcal{H}_{\mathrm{h}}^{1+\beta}$ of radius $\bar{R}_{0}=\sqrt{2 \bar{c}}\|\boldsymbol{f}\|$. In particular,

$$
\overline{\mathcal{B}}_{0}=\left\{U=(\boldsymbol{w}, \eta) \in \mathcal{H}_{\mathrm{h}}^{1+\beta}: \int_{0}^{1}\|U(s)\|_{\mathcal{H}_{\mathrm{h}}^{1+\beta}}^{2} d s \leq 2 \bar{c}\|\boldsymbol{f}\|^{2}\right\}
$$

where $\bar{c}>0$ is a suitable constant.

Let us now consider higher order estimates for the system (28)-(30), taking initial data in $\mathcal{H}_{\mathrm{h}}^{1+2 \beta}$.

Lemma 5.5 Assume $f \in \mathrm{L}^{2}\left(0,+\infty ; \mathrm{H}^{\beta}\right)$. For every initial datum $U_{0}=\left(\boldsymbol{w}_{0}, \eta_{0}\right) \in \mathcal{B}_{0} \subset$ $\mathcal{H}_{\mathrm{h}}^{1+2 \beta}$, we have the estimate

$$
\left\|S(t) U_{0}\right\|_{\mathcal{H}_{\mathrm{h}}^{1+2 \beta}} \leq C, \text { and } \int_{0}^{+\infty}\left\|S(t) U_{0}\right\|_{\mathcal{H}_{\mathrm{h}}^{1+3 \beta}}^{2} d t \leq C .
$$

Proof Testing (28) against $\Lambda^{4 \beta} \boldsymbol{w}$, and (29) against $\Lambda^{4 \beta} \eta$, we find

$$
\begin{aligned}
& \frac{1}{2} \frac{d}{d t}\left(\left\|\Lambda^{2 \beta} \boldsymbol{w}\right\|^{2}+\left\|\nabla_{\mathrm{h}} \Lambda^{2 \beta} \boldsymbol{w}\right\|^{2}\right)+v\left(\left\|\Lambda^{3 \beta} \boldsymbol{w}\right\|^{2}+\alpha^{2}\left\|\nabla_{\mathrm{h}} \Lambda^{3 \beta} \boldsymbol{w}\right\|^{2}\right) \\
& \quad+\int_{0}^{\infty} \mu(s)\left\langle\Lambda^{2 \beta} \eta, \Lambda^{2 \beta} \boldsymbol{w}\right\rangle_{\mathrm{V}_{\mathrm{h}}} d s \\
& \quad \leq\left\|\Lambda^{\beta} \boldsymbol{f}\right\|\left\|\Lambda^{3 \beta} \boldsymbol{w}\right\|+\left|\left(\Lambda^{\beta}((\boldsymbol{w} \cdot \nabla) \boldsymbol{w}), \Lambda^{3 \beta} \boldsymbol{w}\right)\right|,
\end{aligned}
$$

and

$$
\frac{1}{2} \frac{d}{d t}\left\|\Lambda^{2 \beta} \eta\right\|_{\mathcal{M}_{\mathrm{h}}^{1}}^{2}-\int_{0}^{\infty} \mu(s)\left\langle\Lambda^{2 \beta} \eta, \Lambda^{2 \beta} \boldsymbol{w}\right\rangle_{\mathrm{V}_{\mathrm{h}}} d s-\left\langle T \Lambda^{2 \beta} \eta, \Lambda^{2 \beta} \eta\right\rangle_{\mathcal{M}_{\mathrm{h}}^{1}}=0 .
$$

Hence, summing them up we infer

$$
\begin{aligned}
\frac{d}{d t} & \left(\left\|\Lambda^{2 \beta} \boldsymbol{w}\right\|^{2}+\alpha^{2}\left\|\nabla_{\mathrm{h}} \Lambda^{2 \beta} \boldsymbol{w}\right\|^{2}+\left\|\Lambda^{2 \beta} \eta\right\|_{\mathcal{M}_{\mathrm{h}}^{1}}^{2}\right) \\
& +v\left(\left\|\Lambda^{3 \beta} \boldsymbol{w}\right\|^{2}+\alpha^{2}\left\|\nabla_{\mathrm{h}} \Lambda^{3 \beta} \boldsymbol{w}\right\|^{2}\right)-\left\langle T \Lambda^{2 \beta} \eta, \Lambda^{2 \beta} \eta\right\rangle_{\mathcal{M}_{\mathrm{h}}^{1}} \\
& \leq c_{\varepsilon}\left\|\Lambda^{\beta} \boldsymbol{f}\right\|^{2}+\varepsilon\left\|\Lambda^{3 \beta} \boldsymbol{w}\right\|^{2}+\left|\left(\Lambda^{\beta}((\boldsymbol{w} \cdot \nabla) \boldsymbol{w}), \Lambda^{3 \beta} \boldsymbol{w}\right)\right|,
\end{aligned}
$$

and so

$$
\begin{aligned}
\frac{d}{d t} & \left(\left\|\Lambda^{2 \beta} \boldsymbol{w}\right\|^{2}+\alpha^{2}\left\|\nabla_{\mathrm{h}} \Lambda^{2 \beta} \boldsymbol{w}\right\|^{2}+\left\|\Lambda^{2 \beta} \eta\right\|_{\mathcal{M}_{\mathrm{h}}^{1}}^{2}\right) \\
& +v\left(\left\|\Lambda^{3 \beta} \boldsymbol{w}\right\|^{2}+\alpha^{2}\left\|\nabla_{\mathrm{h}} \Lambda^{3 \beta} \boldsymbol{w}\right\|^{2}\right)+\delta\left\|\Lambda^{2 \beta} \eta\right\|_{\mathcal{M}_{\mathrm{h}}^{1}}^{2} \\
& \leq c_{\varepsilon}\left\|\Lambda^{\beta} \boldsymbol{f}\right\|^{2}+\varepsilon\left\|\Lambda^{3 \beta} \boldsymbol{w}\right\|^{2}+\left|\left(\Lambda^{\beta}((\boldsymbol{w} \cdot \nabla) \boldsymbol{w}), \Lambda^{3 \beta} \boldsymbol{w}\right)\right| .
\end{aligned}
$$

Now, we have that

$$
\begin{aligned}
\left|\left(\Lambda^{\beta}((\boldsymbol{w} \cdot \nabla) \boldsymbol{w}), \Lambda^{3 \beta} \boldsymbol{w}\right)\right| & \leq\left\|\Lambda^{\beta}((\boldsymbol{w} \cdot \nabla) \boldsymbol{w})\right\|\left\|\Lambda^{3 \beta} \boldsymbol{w}\right\| \\
& \leq c_{\varepsilon}\left\|\Lambda^{\beta}((\boldsymbol{w} \cdot \nabla) \boldsymbol{w})\right\|^{2}+\varepsilon\left\|\Lambda^{3 \beta} \boldsymbol{w}\right\|^{2},
\end{aligned}
$$


and

$$
\begin{aligned}
& \left\|\Lambda^{\beta}((\boldsymbol{w} \cdot \nabla) \boldsymbol{w})\right\|^{2} \\
& \left.\quad \leq c \| \Lambda^{\beta}\left(\boldsymbol{w}_{\mathrm{h}} \cdot \nabla_{\mathrm{h}}\right) \boldsymbol{w}\right)\left\|^{2}+c\right\| \Lambda^{\beta}\left(w_{3} \partial_{3} \boldsymbol{w}\right) \|^{2} \\
& \quad \leq c\left(\left\|\Lambda^{\beta} \boldsymbol{w}_{\mathrm{h}}\right\|_{\mathrm{L}^{4}}^{2}\left\|\nabla_{\mathrm{h}} \boldsymbol{w}\right\|_{\mathrm{L}^{4}}^{2}+\left\|\boldsymbol{w}_{\mathrm{h}}\right\|_{\mathrm{L}^{\infty}}^{2}\left\|\nabla_{\mathrm{h}} \Lambda^{\beta} \boldsymbol{w}\right\|^{2}\right)+c\left\|w_{3}\right\|_{\mathrm{H}^{1+2 \beta}}^{2}\left\|\partial_{3} \boldsymbol{w}\right\|_{\mathrm{H}^{-1+\beta}}^{2} \\
& \quad \leq c\left(\left\|\Lambda^{2 \beta} \boldsymbol{w}_{\mathrm{h}}\right\|^{2}\left\|\nabla_{\mathrm{h}} \Lambda^{\beta} \boldsymbol{w}\right\|^{2}+\left\|\Lambda^{2 \beta} \boldsymbol{w}_{\mathrm{h}}\right\|^{2}\left\|\nabla_{\mathrm{h}} \Lambda^{\beta} \boldsymbol{w}\right\|^{2}\right)+c\left\|\Lambda^{2 \beta} \nabla w_{3}\right\|^{2}\left\|\Lambda^{\beta} \boldsymbol{w}\right\|^{2}, \\
& \quad \leq c\left\|\Lambda^{2 \beta} \boldsymbol{w}\right\|^{2}\left\|\nabla_{\mathrm{h}} \Lambda^{\beta} \boldsymbol{w}\right\|^{2}+c\left\|\Lambda^{2 \beta} \boldsymbol{w}\right\|^{2}\left\|\nabla_{\mathrm{h}} \Lambda^{\beta} \boldsymbol{w}\right\|^{2}+c\left\|\Lambda^{2 \beta} \nabla_{\mathrm{h}} \boldsymbol{w}\right\|^{2}\left\|\Lambda^{\beta} \boldsymbol{w}\right\|^{2},
\end{aligned}
$$

where we used the embeddings $\mathrm{W}^{2 \beta, 2} \hookrightarrow \mathrm{L}^{\infty}, \mathrm{W}^{\beta, 2} \hookrightarrow \mathrm{L}^{4}$, Lemma 2.1 with $p_{1}=q_{1}=4$, and $p_{2}=\infty, q_{2}=2$, and Lemma 2.2 with $s_{0}=-\beta, s_{1}=1+2 \beta$, and $s_{2}=-1+\beta$ (and $\beta \geq 3 / 4)$.

Therefore, using the above estimates along with (86) and (87), we infer

$$
\begin{aligned}
& \frac{d}{d t}\left(\left\|\Lambda^{2 \beta} \boldsymbol{w}\right\|^{2}+\alpha^{2}\left\|\nabla_{\mathrm{h}} \Lambda^{2 \beta} \boldsymbol{w}\right\|^{2}+\left\|\Lambda^{2 \beta} \eta\right\|_{\mathcal{M}_{\mathrm{h}}^{1}}^{2}\right)+(v-2 \varepsilon)\left(\left\|\Lambda^{3 \beta} \boldsymbol{w}\right\|^{2}+\alpha^{2}\left\|\nabla_{\mathrm{h}} \Lambda^{3 \beta} \boldsymbol{w}\right\|^{2}\right) \\
& \quad+\delta\left\|\Lambda^{2 \beta} \eta\right\|_{\mathcal{M}_{\mathrm{h}}^{1}}^{2} \\
& \quad \leq c_{\varepsilon}\left\|\Lambda^{\beta} \boldsymbol{f}\right\|^{2}+C\left(\left\|\Lambda^{\beta} \boldsymbol{w}\right\|^{2}+\left\|\nabla_{\mathrm{h}} \Lambda^{\beta} \boldsymbol{w}\right\|^{2}\right)\left(\left\|\Lambda^{2 \beta} \boldsymbol{w}\right\|^{2}+\left\|\nabla_{\mathrm{h}} \Lambda^{2 \beta} \boldsymbol{w}\right\|^{2}\right) .
\end{aligned}
$$

Integrating in time, we reach and using Gronwall's inequality we obtain

$$
\begin{aligned}
& \left(\left\|\Lambda^{2 \beta} \boldsymbol{w}\right\|^{2}+\alpha^{2}\left\|\nabla_{\mathrm{h}} \Lambda^{2 \beta} \boldsymbol{w}\right\|^{2}+\left\|\Lambda^{2 \beta} \eta\right\|_{\mathcal{M}_{\mathrm{h}}^{1}}^{2}\right)(t) \\
& \quad+\hat{v} \int_{0}^{t}\left(\left\|\Lambda^{3 \beta} \boldsymbol{w}\right\|^{2}+\alpha^{2}\left\|\nabla_{\mathrm{h}} \Lambda^{3 \beta} \boldsymbol{w}\right\|^{2}+\left\|\Lambda^{2 \beta} \eta\right\|_{\mathcal{M}_{\mathrm{h}}^{1}}^{2}\right) d s \\
& \quad \leq\left(\left\|\Lambda^{2 \beta} \boldsymbol{w}_{0}\right\|^{2}+\alpha^{2}\left\|\nabla_{\mathrm{h}} \Lambda^{2 \beta} \boldsymbol{w}_{0}\right\|^{2}+\left\|\Lambda^{2 \beta} \eta_{0}\right\|_{\mathcal{M}_{\mathrm{h}}^{1}}^{2}+c \int_{0}^{+\infty}\left\|\Lambda^{\beta} \boldsymbol{f}\right\|^{2} d s\right) e^{\int_{0}^{t} \overline{\mathbf{b}}(s) d s},
\end{aligned}
$$

with $\overline{\mathbf{b}}=\left(\left\|\Lambda^{\beta} \boldsymbol{w}\right\|^{2}+\left\|\Lambda^{\beta} \nabla_{\mathrm{h}} \boldsymbol{w}\right\|^{2}\right) \in \mathrm{L}^{2}(0,+\infty)$, as a consequence of Remark 3.4. Thanks to this last estimate the proof is concluded.

\subsection{Further Estimates for $w_{t}$}

Let us assume $\left(\boldsymbol{w}_{0}, \eta_{0}\right) \in \mathcal{H}_{\mathrm{h}}^{1+\beta}=\mathrm{V}_{\mathrm{h}} \times \mathcal{M}_{\mathrm{h}}^{1}$ and $\boldsymbol{f}=\boldsymbol{f}(x)$. Then testing (28) against $\boldsymbol{w}_{t}$, in $\mathrm{L}^{2}$, using Hölder's and Young's inequalities and properly reabsorbing the so obtained terms on the left-hand side, we get

$$
\begin{aligned}
& (1-\varepsilon)\left(\left\|\boldsymbol{w}_{t}\right\|^{2}+\alpha^{2}\left\|\nabla_{\mathrm{h}} \boldsymbol{w}_{t}\right\|^{2}\right) \leq c_{\varepsilon}\left(v\left(\|\boldsymbol{w}\|^{2}+\alpha^{2}\left\|\nabla_{\mathrm{h}} \Lambda^{\beta} \boldsymbol{w}\right\|^{2}\right)+\|\eta\|_{\mathcal{M}_{\mathrm{h}}^{1}}^{2}+\|\boldsymbol{f}\|^{2}\right) \\
& \quad+c_{\varepsilon}\|(\boldsymbol{w} \cdot \nabla) \boldsymbol{w}\|^{2},
\end{aligned}
$$

and it holds that

$$
\begin{aligned}
\|\boldsymbol{w} \cdot \nabla \boldsymbol{w}\|^{2} & \leq c\left\|\boldsymbol{w}_{\mathrm{h}} \cdot \nabla_{\mathrm{h}} \boldsymbol{w}\right\|^{2}+c\left\|w_{3} \partial_{3} \boldsymbol{w}\right\|^{2} \\
& \leq c\left\|\boldsymbol{w}_{\mathrm{h}}\right\|_{\mathrm{L}^{4}}^{2}\left\|\nabla_{\mathrm{h}} \boldsymbol{w}\right\|_{\mathrm{L}^{4}}^{2}+c\left\|w_{3}\right\|_{\mathrm{H}^{1+\beta}}^{2}\left\|\partial_{3} \boldsymbol{w}\right\|_{\mathrm{H}^{-1+\beta}}^{2} \\
& \left.\leq c\left\|\Lambda^{\beta} \boldsymbol{w}_{\mathrm{h}}\right\|^{2}\left\|\Lambda^{\beta} \nabla_{\mathrm{h}} \boldsymbol{w}\right\|^{2}+c\left\|\Lambda^{\beta} \nabla w_{3}\right\|^{2}\left\|\Lambda^{\beta} \boldsymbol{w}\right\|^{2}\right) \\
& \leq C\left(\left\|\Lambda^{\beta} \boldsymbol{w}_{\mathrm{h}}\right\|^{2}\left\|\Lambda^{\beta} \nabla_{\mathrm{h}} \boldsymbol{w}\right\|^{2}+\left\|\Lambda^{\beta} \nabla_{\mathrm{h}} \boldsymbol{w}_{\mathrm{h}}\right\|^{2}\left\|\Lambda^{\beta} \boldsymbol{w}\right\|^{2}\right) \\
& \leq C\left\|\Lambda^{\beta} \boldsymbol{w}\right\|^{2}\left\|\Lambda^{\beta} \nabla_{\mathrm{h}} \boldsymbol{w}\right\|^{2},
\end{aligned}
$$


where, up to lower order terms, we used Lemma 2.2 with $s_{0}=0, s_{1}=1+\beta$, and $s_{2}=-1+\beta$ (and $\beta \geq 3 / 4$ ). In particular, from (71), we have that $\left\|\Lambda^{\beta} \boldsymbol{w}\right\|,\left\|\Lambda^{\beta} \nabla_{\mathrm{h}} \boldsymbol{w}\right\| \in \mathrm{L}_{\mathrm{loc}}^{\infty}(0,+\infty)$. As a direct consequence of the above estimates, we obtain

$$
\int_{0}^{t}\left(\left\|\boldsymbol{w}_{t}\right\|^{2}+\alpha^{2}\left\|\nabla_{\mathrm{h}} \boldsymbol{w}_{t}\right\|^{2}\right) d s \leq C(1+t),
$$

and so it holds that $\left\|\boldsymbol{w}_{t}\right\|, \alpha\left\|\nabla_{\mathrm{h}} \boldsymbol{w}_{t}\right\| \in \mathrm{L}_{\mathrm{loc}}^{2}(0,+\infty)$.

In the same spirit as before, if we assume $\left(\boldsymbol{w}_{0}, \eta_{0}\right) \in \mathcal{H}_{\mathrm{h}}^{1+2 \beta}$ and $\boldsymbol{f} \in \mathrm{H}^{\beta}$, then we can prove that $\left\|\Lambda^{\beta} \boldsymbol{w}_{t}\right\|, \alpha\left\|\nabla_{\mathrm{h}} \Lambda^{\beta} \boldsymbol{w}_{t}\right\| \in \mathrm{L}_{\text {loc }}^{2}(0,+\infty)$. Indeed, testing (28) against $\Lambda^{2 \beta} \boldsymbol{w}_{t}$, in $L^{2}$, using Hölder's and Young's inequalities and properly reabsorbing the so obtained terms on the left-hand side, we get

$$
\begin{aligned}
& (1-\varepsilon)\left(\left\|\Lambda^{\beta} \boldsymbol{w}_{t}\right\|^{2}+\alpha^{2}\left\|\nabla_{\mathrm{h}} \Lambda^{\beta} \boldsymbol{w}_{t}\right\|^{2}\right) \\
& \quad \leq c_{\varepsilon}\left(v\left(\left\|\Lambda^{2 \beta} \boldsymbol{w}\right\|^{2}+\alpha^{2}\left\|\nabla_{\mathrm{h}} \Lambda^{2 \beta} \boldsymbol{w}\right\|^{2}\right)+\left\|\Lambda^{\beta} \eta\right\|_{\mathcal{M}_{\mathrm{h}}^{1}}^{2}+\left\|\Lambda^{\beta} \boldsymbol{f}\right\|^{2}\right) \\
& \quad+c_{\varepsilon}\left\|\Lambda^{\beta}((\boldsymbol{w} \cdot \nabla) \boldsymbol{w})\right\|^{2} .
\end{aligned}
$$

It is enough to control the nonlinear term. Hence, with the same identical calculations as done in Lemma 5.5, we have

$$
\begin{aligned}
& c_{\varepsilon}\left\|\Lambda^{\beta}((\boldsymbol{w} \cdot \nabla) \boldsymbol{w})\right\|^{2} \leq c\left\|\Lambda^{2 \beta} \boldsymbol{w}_{\mathrm{h}}\right\|^{2}\left\|\nabla_{\mathrm{h}} \Lambda^{\beta} \boldsymbol{w}\right\|^{2}+c\left\|\nabla_{\mathrm{h}} \Lambda^{\beta} \boldsymbol{w}_{\mathrm{h}}\right\|^{2}\left\|\Lambda^{2 \beta} \boldsymbol{w}\right\|^{2} \\
& \quad+c\left\|\Lambda^{2 \beta} \nabla_{\mathrm{h}} \boldsymbol{w}_{\mathrm{h}}\right\|^{2}\left\|\Lambda^{\beta} \boldsymbol{w}\right\|^{2} .
\end{aligned}
$$

Using (91) together with the above control, we obtain the claimed regularity for $\boldsymbol{w}_{t}$.

\section{Exponentially Attracting Sets}

It is known that dynamical systems generated by equation with memory do not regularize in finite time, due to the nature of the memory term (see, e.g., [19]). This behavior still remain the same even in the case under consideration. In particular, this prevents the existence of absorbing sets having higher regularity than the initial data.

Definition 6.1 A bounded set $\mathcal{B}_{\star}$ is said to be exponentially attracting for $S(t)$ in $\mathcal{H}_{\mathrm{h}}^{1+\beta}$ if there exists $\omega>0$ such that

$$
\operatorname{dist}_{\mathcal{H}_{\mathrm{h}}^{1+\beta}}\left(S(t) B, \mathcal{B}_{\star}\right) \leq \mathcal{Q}\left(\|B\|_{\mathcal{H}_{\mathrm{h}}^{1+\beta}}\right) e^{-\omega t} \text { for every bounded subset } B \subset \mathcal{H}_{\mathrm{h}}^{1+\beta},
$$

where $\mathcal{Q}$ is a generic positive increasing function, and

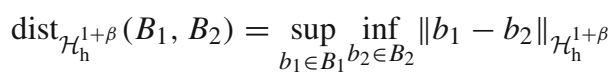

is the Hausdorff semidistance in $\mathcal{H}_{\mathrm{h}}^{1+\beta}$ between two (nonempty) sets $B_{1}$ and $B_{2}$.

Proposition 6.2 There exists $R_{\star}>0$ such that the ball

$$
\mathcal{B}_{\star}:=\mathrm{B}_{\mathcal{H}_{\mathrm{h}}^{1+\beta}}\left(R_{\star}\right)
$$

is exponentially attracting for $S(t)$. 
It is enough to prove that the ball $\mathcal{B}_{\star}$ exponentially attracts the absorbing set $\overline{\mathcal{B}}_{0} \subset \mathcal{H}_{\mathrm{h}}^{1+\beta}$ determined in (46), Sect. 5. To this end, for every initial data $U_{0}=\left(\boldsymbol{w}_{0}, \eta_{0}\right) \in \overline{\mathcal{B}}_{0}$, in the same spirit of Temam [35] we decompose the solution $S(t) U_{0}$ into the sum

$$
S(t) U_{0}=L(t) U_{0}+K(t) U_{0},
$$

where $L(t) U_{0}=(\boldsymbol{v}(t), \xi(t))$ and $K(t) U_{0}=(\boldsymbol{u}(t), \zeta(t))$ solve, respectively, the problems

$$
\begin{aligned}
& \partial_{t}\left(I-\alpha^{2} \Delta_{\mathrm{h}}\right) \boldsymbol{v}-v \Lambda^{2 \beta}\left(I-\alpha^{2} \Delta_{\mathrm{h}}\right) \boldsymbol{v}-\int_{0}^{\infty} \mu(s) A_{\mathrm{h}} \xi(s) d s=0, \\
& \partial_{t} \xi=T \xi+\boldsymbol{v}, \\
& (\boldsymbol{v}(0), \xi(0))=\left(\boldsymbol{w}_{0}, \eta_{0}\right),
\end{aligned}
$$

and

$$
\begin{aligned}
& \partial_{t}\left(I-\alpha^{2} \Delta_{\mathrm{h}}\right) \mathrm{u}-v \Lambda^{2 \beta}\left(I-\alpha^{2} \Delta_{\mathrm{h}}\right) \mathrm{u}-\int_{0}^{\infty} \mu(s) A_{\mathrm{h}} \zeta(s) d s+(\boldsymbol{w} \cdot \nabla) \mathrm{u}=\tilde{\boldsymbol{f}}, \\
& \partial_{t} \zeta=T \zeta+\mathrm{u}, \\
& (\mathrm{u}(0), \zeta(0))=(0,0),
\end{aligned}
$$

with $\widetilde{f}=\boldsymbol{f}-(\boldsymbol{w} \cdot \nabla) \boldsymbol{v}$.

Existence and uniqueness of these problems are similar to the case previously considered for the full system (28)-(30). We will show that system (94) is exponentially stable with respect the $\mathcal{H}_{\mathrm{h}}^{1+\beta}$-norm, and that the solutions to (95) are uniformly bounded in the space $\mathcal{H}_{\mathrm{h}}^{1+2 \beta}$.

In the sequel, the generic positive constant $C$ may depend on $\|f\|$ as well as on the radius $\bar{R}_{0}$ of the absorbing set $\overline{\mathcal{B}}_{0}$. The proof of Proposition 6.2 is a direct consequence consequence of Lemma 6.3 and Lemma 6.4 here below.

Lemma 6.3 The following control holds true

$$
\left\|L(t) U_{0}\right\|_{\mathcal{H}_{\mathrm{h}}^{1+\beta}} \leq c e^{-\omega t},
$$

where $\omega:=\varepsilon(\varrho-C \varepsilon \gamma)$, with $\varepsilon$ and $\gamma$ are utility constants, $\varepsilon$ as needed, and $\varrho=\varrho(\nu, \alpha)$.

Observe that, control (96) (and hence Lemma 6.3) can be proved by following the same steps used to reach (73). We are now ready to show that the solutions of (95) are uniformly bounded in $\mathcal{H}_{\mathrm{h}}^{1+\beta}$

Lemma 6.4 Assume $f \in \mathrm{L}^{2}\left(0,+\infty ; \mathrm{L}^{2}\right)$. For every initial datum $U_{0} \in \mathcal{B}_{0} \subset \mathcal{H}_{\mathrm{h}}^{1+\beta}$, we have the estimate

$$
\left\|K(t) U_{0}\right\|_{\mathcal{H}_{\mathrm{h}}^{1+2 \beta}} \leq C \text {, and } \int_{0}^{+\infty}\left\|K(t) U_{0}\right\|_{\mathcal{H}_{\mathrm{h}}^{1+3 \beta}}^{2} d t \leq C .
$$

Also in this case, the above result follows by the same calculations previously performed to reach (83).

We highlight the fact that we do not expect compactness for the embedding $\mathcal{M}_{\mathrm{h}}^{1+2 \beta} \subset$ $\mathcal{M}_{\mathrm{h}}^{1+\beta}$ (see [29] for a counterexample to compactness in the case of isotropic spaces with memory). As a consequence, also the embedding $\mathcal{H}_{\mathrm{h}}^{1+2 \beta} \subset \mathcal{H}_{\mathrm{h}}^{1+\beta}$ is in general not compact as well. However, there is a general argument (see [29]) that allows to retrieve the desired compactness, producing a compact subset $\mathcal{B}_{\star}^{\prime} \subset \mathcal{B}_{\star}$ which is still exponentially attracting. In turn, thanks to well-established results, this entails the existence of the global attractor $\mathcal{A}$. 
Remark 6.5 After obtaining the higher-order energy estimates in Sect. 5 (used directly to prove Lemmas 6.3 and 6.4 ), and proving the existence of an absorbing set in $\mathcal{H}_{\mathrm{h}}^{1+\beta}$, to show the existence of a global exponential attractor $\mathfrak{E} \subset \mathcal{H}_{\mathrm{h}}^{1+\beta}$ for the system (28)-(30), we adapt the proof of [19, Theorem 7.2], since, at this level, the main technical difficulty left is related to the presence of the memory term.

\section{Exponential Attractor}

In this section we establish the existence of a regular exponential attractor for the semigroup associated with system (28)-(30) in the phase space $\mathcal{H}_{\mathrm{h}}^{1+\beta}$.

For the reader's convenience, we recall the definition of exponential attractor (see also $[3,19,29])$. In the next definition we consider a general $\mathcal{H}$ that, however, in our case, plays the role of $\mathcal{H}_{\mathrm{h}}^{1+\beta}$.

Definition 7.1 Consider a semiflow $S(t): \mathcal{H} \rightarrow \mathcal{H}, t \geq 0$, on the phase space $\mathcal{H}$. A compact set $\mathfrak{E} \subset \mathcal{H}$ is an exponential attractor for $S(t)$ if

- $\mathfrak{E}$ is positively invariant, i.e. $S(t) \mathfrak{E} \subset \mathfrak{E}$ for every $t \geq 0$,

- $\mathfrak{E}$ is exponentially attracting for the semigroup,

- $\mathfrak{E}$ has finite fractal dimension in $\mathcal{H}$.

Let us also recall that the fractal dimension of $\mathfrak{E}$ in $\mathcal{H}$ is defined as

$$
\operatorname{dim}_{\mathcal{H}}(\mathfrak{E})=\limsup _{\varepsilon \rightarrow 0} \frac{\ln N(\varepsilon)}{\ln (1 / \varepsilon)},
$$

where $N(\varepsilon)$ is the smallest number of $\varepsilon$-balls of $\mathcal{H}$ needed to cover $\mathfrak{E}$.

The main result of the paper reads as follows.

Theorem 7.2 Assume $\beta \geq 3 / 4$ and $f \in \mathrm{L}^{2}\left(0,+\infty, \mathrm{L}^{2}\right) \cap \mathrm{L}_{\text {loc }}^{2}\left(0,+\infty, \mathrm{H}^{\beta}\right)$. The dynamical system $S(t)$ on $\mathcal{H}_{\mathrm{h}}^{1+\beta}$ admits an exponential attractor $\mathfrak{E}$, which is bounded in $\mathcal{H}_{\mathrm{h}}^{1+2 \beta}$.

As a consequence of the existence of a compact attracting set, $S(t)$ possesses the global attractor $\mathcal{A}$, which is the smallest among the compact attracting sets (hence contained in the exponential attractor).

COROLlario The dynamical system $S(t)$ on $\mathcal{H}_{\mathrm{h}}^{1+\beta}$ possesses the global attractor $\mathcal{A}$.

The Proof of Theorem 7.2, carried out in the next Subsection, is based on an abstract result from Danese et al. (2015) (see Theorem 5.1, see also Lemma), that we report here below as a lemma, in a specific version prepared to fit our particular case. To this end, we will make use of the projections $\mathbb{P}_{1}$ and $\mathbb{P}_{2}$ of $\mathcal{H}_{\mathrm{h}}^{1+\beta}$ onto its components $\mathrm{H}_{\mathrm{h}}^{1+\beta}$ and $\mathcal{M}_{\mathrm{h}}^{1+\beta}$, namely,

$$
\mathbb{P}_{1}(u, \eta)=u \text { and } \mathbb{P}_{2}(u, \eta)=\eta \text {. }
$$

Lemma 7.3 Let the following assumptions hold.

(i) There exists $R_{\star}>0$ such that the ball $B_{\star}=\mathrm{B}_{\mathcal{H}^{1+2 \beta}}\left(R_{\star}\right)$ is exponentially attracting.

(ii) Assume For every $R \geq 0$ and every $\theta>0$ sufficlently large, then

$$
\int_{\theta}^{2 \theta}\left(\left\|\Lambda^{\beta} \boldsymbol{w}_{t}\right\|^{2}+\alpha^{2}\left\|\nabla_{\mathrm{h}} \Lambda^{\beta} \boldsymbol{w}_{t}\right\|^{2}\right) d s \leq \mathcal{Q}(R+\theta) .
$$

for all $\boldsymbol{w}(t)=\mathbb{P}_{1} S(t) U_{0}$ with $U_{0} \in \mathrm{B}_{\mathcal{H}_{\mathrm{h}}^{1+2 \beta}}(R)$. 
(iii) There exists $R_{1}>0$ such that: For any given $R \geq 0$, there exists a nonnegative function $\psi$ vanishing at infinity such that

$$
\left\|S(t) U_{0}\right\|_{\mathcal{H}_{\mathrm{h}}^{1+2 \beta}} \leq \psi(t)+R_{1},
$$

for all $U_{0} \in \mathrm{B}_{\mathcal{H}_{\mathrm{h}}^{1+2 \beta}}(R)$.

(iv) For every fixed $R \geq 0$, the semigroup $S(t)$ admits a decomposition of the form $S(t)=$ $L(t)+K(t)$ satisfying for all initial data $U_{0, i} \in \mathrm{B}_{\mathcal{H}_{\mathrm{h}}^{1+\beta}(R)}$ relations

$$
\begin{aligned}
& \left\|L(t) U_{0,1}-L(t) U_{0,2}\right\|_{\mathcal{H}_{\mathrm{h}}^{1+\beta}} \leq \psi(t)\left\|U_{0,1}-U_{0,2}\right\|_{\mathcal{H}_{\mathrm{h}}^{1+\beta}}, \\
& \left\|K(t) U_{0,1}-K(t) U_{0,2}\right\|_{\mathcal{H}_{\mathrm{h}}^{1+2 \beta}} \leq \mathcal{Q}(t)\left\|U_{0,1}-U_{0,2}\right\|_{\mathcal{H}_{\mathrm{h}}^{1+\beta}} .
\end{aligned}
$$

Here, both $\mathcal{Q}$ and the nonnegative function $\psi$ vanishing at infinity depend on $R$. Moreover, the function

$$
\bar{\zeta}^{t}=\mathbb{P}_{2} K(t) U_{0,1}-\mathbb{P}_{2} K(t) U_{0,2}
$$

satisfies the Cauchy problem

$$
\left\{\begin{array}{l}
\partial_{t} \bar{\zeta}^{t}=T \bar{\zeta}^{t}+\bar{w}(t) \\
\bar{\zeta}_{0}=0
\end{array}\right.
$$

for some $\bar{w}$ satisfying the estimate $\|\bar{w}(t)\|_{\mathrm{H}_{\mathrm{h}}^{1+\beta}} \leq \mathcal{Q}(t)\left\|U_{0,1}-U_{0,2}\right\|_{\mathcal{H}_{\mathrm{h}}^{1+\beta}}$.

Then $S(t)$ possesses an exponential attractor $\mathfrak{E}$ contained in the ball $\mathrm{B}_{\mathcal{H}_{\mathrm{h}}^{1+2 \beta}}\left(R_{1}\right)$.

Remark 7.4 The above result is a consequence of [19, Lemma 7.4] (see also [35]) which has been adapted to the present framework.

\subsection{Proof of Theorem 7.2}

Essentially, we have to prove the four points in Lemma 7.3. We actually have that: point (i) is the content of Proposition 6.2, while (ii) is an immediate consequence of relations (91)-(92). Accordingly, we are left to show the validity of (iii) and (iv). In what follows, the generic positive constant $C$ may depend on $\|\boldsymbol{f}\|$ (or on $\|\boldsymbol{f}\|_{\mathrm{H}^{\beta}}$ ) and on the radius $\bar{R}_{0}$ of the absorbing set $\overline{\mathcal{B}}_{0}$ defined in (82).

-Point (iii): Given $R \geq 0$, let us consider the ball $\mathrm{B}_{\mathcal{H}_{\mathrm{h}}^{1+2 \beta}}(R)$. We easily infer from the continuous embedding $\mathcal{H}_{\mathrm{h}}^{1+2 \beta} \subset \mathcal{H}_{\mathrm{h}}^{1+\beta}$ that $\mathrm{B}_{\mathcal{H}_{\mathrm{h}}^{1+2 \beta}}(R) \subset \mathrm{B}_{\mathcal{H}_{\mathrm{h}}^{1+\beta}}(\mathcal{Q}(R))$. Therefore, on account of (50),

there exists $t_{e}=t_{e}(R)$ such that

$$
\left\{\begin{array}{l}
\left\|S(t) \mathrm{B}_{\mathcal{H}_{\mathrm{h}}^{1+2 \beta}}(R)\right\| \leq \mathcal{Q}(R), \quad \forall t \leq t_{e} \\
\left\|S(t) \mathrm{B}_{\mathcal{H}_{\mathrm{h}}^{1+2 \beta}}(R)\right\| \leq R_{0}, \quad \forall t \geq t_{e} .
\end{array}\right.
$$

Taking an arbitrary $U_{0} \in \mathrm{B}_{\mathcal{H}_{\mathrm{h}}^{1+2 \beta}}(R)$, we define the higher-order energy functional

$$
\mathcal{E}_{1+2 \beta}(t)=\frac{1}{2}\left\|S(t) U_{0}\right\|_{\mathcal{H}_{\mathrm{h}}^{1+2 \beta}}^{2} .
$$


Now, using (99) and Lemma 5.5, and proceeding as in Lemma 5.3, we obtain point (iii) of Lemma 7.3.

-Point (iv): Now, we refer to the splitting performed in (94) and (95). In this case we have already seen that $L(t)$ is a strongly continuous linear semigroup on $\mathcal{H}_{\mathrm{h}}^{1+\beta}$. Besides, $L(t)$ is exponentially stable. This is a direct consequence of the results proved in the previous section.

Let us now take $R \geq 0$ be fixed, and let $U_{0,1}, U_{0,2} \in B_{\mathcal{H}_{\mathrm{h}}^{1+2 \beta}}(R)$. For the remainder of this proof, the generic positive constant $C$ is allowed to depend on $R$. Then, we decompose the difference Then, we decompose the difference

$$
\left(\overline{\boldsymbol{w}}(t), \bar{\eta}^{t}\right)=S(t) U_{0,1}-S(t) U_{0,2}
$$

into the sum

$$
\left(\overline{\boldsymbol{w}}(t), \bar{\eta}^{t}\right)=\left(\overline{\boldsymbol{v}}(t), \bar{\xi}^{t}\right)+\left(\overline{\mathrm{u}}(t), \bar{\zeta}^{t}\right),
$$

where

$$
\left(\overline{\boldsymbol{v}}(t), \bar{\xi}^{t}\right)=L(t) U_{0,1}-L(t) U_{0,2}, \quad \text { and }\left(\overline{\boldsymbol{u}}(t), \bar{\zeta}^{t}\right)=K(t) U_{0,1}-K(t) U_{0,2} .
$$

Observe that, thanks to relation (iii), the following relation holds true

$$
\left\|S(t) U_{0, i}\right\|_{\mathcal{H}_{\mathrm{h}}^{1+2 \beta}} \leq C .
$$

Moreover, the exponential stability of $L(t)$ implies the existence of a universal constant $\varpi>0$ (see $[19,35])$ such that

$$
\left\|L(t) U_{0,1}-L(t) U_{0,2}\right\|_{\mathcal{H}_{\mathrm{h}}^{1+\beta}} \leq C e^{-\varpi t}\left\|U_{0,1}-U_{0,2}\right\|_{\mathcal{H}_{\mathrm{h}}^{1+\beta}}
$$

Essentially, we have to prove the desired estimate for the difference $(\bar{u}, \bar{\zeta})$, solution to the system

$$
\begin{aligned}
& \partial_{t}\left(I-\alpha^{2} \Delta_{\mathrm{h}}\right) \overline{\mathrm{u}}-v \Lambda^{2 \beta}\left(I-\alpha^{2} \Delta_{\mathrm{h}}\right) \overline{\mathrm{u}}-\int_{0}^{\infty} \mu(s) A_{\mathrm{h}} \bar{\zeta}(s) d s=-(\overline{\boldsymbol{w}} \cdot \nabla) \boldsymbol{w}_{1}-\left(\boldsymbol{w}_{2} \cdot \nabla\right) \overline{\boldsymbol{w}} \\
& \partial_{t} \bar{\zeta}=T \bar{\zeta}+\overline{\mathrm{u}} \\
& \left(\overline{\mathrm{u}}(0), \bar{\zeta}^{0}\right)=(0,0) .
\end{aligned}
$$

Testing (28) against $\Lambda^{4 \beta} \overline{\mathrm{u}}$ in $\mathrm{L}^{2}$ and (29) against $\Lambda^{4 \beta} \bar{\zeta}$ in $\mathcal{M}_{\mathrm{h}}^{1}$, and proceeding as before, we reach

$$
\begin{aligned}
\frac{1}{2} & \frac{d}{d t}\left(\left\|\Lambda^{2 \beta} \overline{\mathrm{u}}\right\|^{2}+\alpha^{2}\left\|\nabla_{\mathrm{h}} \Lambda^{2 \beta} \overline{\mathrm{u}}\right\|^{2}+\left\|\Lambda^{2 \beta} \bar{\zeta}\right\|_{\mathcal{M}_{\mathrm{h}}^{1}}^{2}\right)+v\left(\left\|\Lambda^{3 \beta} \overline{\mathrm{u}}\right\|^{2}+\alpha^{2}\left\|\nabla_{\mathrm{h}} \Lambda^{3 \beta} \overline{\mathrm{u}}\right\|^{2}\right) \\
& -\left\langle T \Lambda^{2 \beta} \bar{\zeta}, \Lambda^{2 \beta} \bar{\zeta}\right\rangle_{\mathcal{M}_{\mathrm{h}}^{1}} \\
& \left.\leq\left|\left(\Lambda^{\beta}\left((\overline{\boldsymbol{w}} \cdot \nabla) \boldsymbol{w}_{1}\right), \Lambda^{3 \beta} \overline{\mathrm{u}}\right)\right|+\mid\left(\Lambda^{\beta}\left(\left(\boldsymbol{w}_{2} \cdot \nabla\right) \overline{\boldsymbol{w}}\right)\right), \Lambda^{3 \beta} \overline{\mathrm{u}}\right) \mid:=\tilde{I}_{1}+\tilde{I}_{2}
\end{aligned}
$$


Then, arguing as in the Proof of Lemma 5.5, we have that

$$
\begin{aligned}
\tilde{I}_{1} \leq & c_{\varepsilon}\left(\left\|\Lambda^{\beta}\left(\left(\overline{\boldsymbol{w}}_{\mathrm{h}} \cdot \nabla_{\mathrm{h}}\right) \boldsymbol{w}_{1}\right)\right\|^{2}+\left\|\Lambda^{\beta}\left(\bar{w}_{3} \partial_{3} \boldsymbol{w}_{1}\right)\right\|^{2}\right)+\varepsilon\left\|\Lambda^{3 \beta} \overline{\mathrm{u}}\right\|^{2} \\
\leq & c\left(\left\|\Lambda^{\beta} \overline{\boldsymbol{w}}_{\mathrm{h}}\right\|^{2}\left\|\nabla_{\mathrm{h}} \boldsymbol{w}_{1}\right\|_{\mathrm{L}^{\infty}}^{2}+\left\|\overline{\boldsymbol{w}}_{\mathrm{h}}\right\|_{\mathrm{L}^{4}}^{2}\left\|\nabla_{\mathrm{h}} \Lambda^{\beta} \boldsymbol{w}_{1}\right\|_{\mathrm{L}^{4}}^{2}\right) \\
& +c\left(\left\|\Lambda^{\beta} \bar{w}_{3}\right\|_{\mathrm{L}^{6}}^{2}\left\|\partial_{3} \boldsymbol{w}_{1}\right\|_{\mathrm{L}^{3}}^{2}+\left\|\bar{w}_{3}\right\|_{\mathrm{L}^{\infty}}^{2}\left\|\partial_{3} \Lambda^{\beta} \boldsymbol{w}_{1}\right\|^{2}\right) \\
& +\varepsilon \| \Lambda^{3 \beta} \overline{\mathrm{u}}^{2} \\
\leq & c\left\|\Lambda^{\beta} \overline{\boldsymbol{w}}_{\mathrm{h}}\right\|^{2}\left\|\nabla_{\mathrm{h}} \Lambda^{2 \beta} \boldsymbol{w}_{1}\right\|^{2}+c\left\|\nabla_{\mathrm{h}} \Lambda^{\beta} \overline{\boldsymbol{w}}_{\mathrm{h}}\right\|^{2}\left\|\Lambda^{2 \beta} \boldsymbol{w}_{1}\right\|^{2} \\
& +c\left\|\Lambda^{\beta} \overline{\boldsymbol{w}}_{\mathrm{h}}\right\|^{2}\left\|\nabla_{\mathrm{h}} \Lambda^{2 \beta} \boldsymbol{w}_{1}\right\|^{2}+c\left\|\Lambda^{\beta} \nabla_{\mathrm{h}} \overline{\boldsymbol{w}}_{\mathrm{h}}\right\|^{2}\left\|\boldsymbol{w}_{1}\right\|_{\mathrm{H}^{3 \beta}}^{2}+\varepsilon\left\|\Lambda^{3 \beta} \overline{\mathrm{u}}\right\|^{2} \\
\leq & C\left(\left\|\Lambda^{\beta} \overline{\mathrm{u}}\right\|^{2}+\left\|\nabla_{\mathrm{h}} \Lambda^{\beta} \overline{\mathrm{u}}\right\|^{2}\right)\left(\left\|\Lambda^{3 \beta} \boldsymbol{w}_{1}\right\|^{2}+\left\|\nabla_{\mathrm{h}} \Lambda^{2 \beta} \boldsymbol{w}_{1}\right\|^{2}\right. \\
& \left.+\left\|\Lambda^{2 \beta} \boldsymbol{w}_{1}\right\|^{2}+\left\|\nabla_{\mathrm{h}} \Lambda^{\beta} \boldsymbol{w}_{1}\right\|^{2}\right)+C\left(\left\|\Lambda^{\beta} \overline{\boldsymbol{v}}\right\|^{2}+\left\|\nabla_{\mathrm{h}} \Lambda^{\beta} \overline{\boldsymbol{v}}\right\|^{2}\right)\left(\left\|\Lambda^{3 \beta} \boldsymbol{w}_{1}\right\|^{2}\right. \\
& \left.+\left\|\nabla_{\mathrm{h}} \Lambda^{2 \beta} \boldsymbol{w}_{1}\right\|^{2}+\left\|\Lambda^{2 \beta} \boldsymbol{w}_{1}\right\|^{2}+\left\|\nabla_{\mathrm{h}} \Lambda^{\beta} \boldsymbol{w}_{1}\right\|^{2} \|\right)+\varepsilon \| \Lambda^{3 \beta} \overline{\mathrm{u}}^{2},
\end{aligned}
$$

where we used Lemma 2.1 with $p_{1}=q_{1}=4$ and $p_{2}=\infty$ and $q_{2}=2$ for the first term, then $p_{1}=\infty$, and $q_{1}=2$ and $p_{2}=6$ and $q_{2}=3$ for the second term, and the embeddings $\mathrm{W}^{3 \beta, 2} \hookrightarrow \mathrm{W}^{1+\beta, 2}, \mathrm{~W}^{2 \beta, 2} \hookrightarrow \mathrm{L}^{\infty}, \mathrm{W}^{1+\beta, 2} \hookrightarrow \mathrm{L}^{\infty}, \mathrm{W}^{2 \beta, 2} \hookrightarrow \mathrm{W}^{1,3}, \mathrm{~W}^{\beta, 2} \hookrightarrow$ $\mathrm{L}^{4}$, and $\mathrm{H}^{1} \hookrightarrow \mathrm{L}^{6}$. Observe that $\left\|\Lambda^{3 \beta} \boldsymbol{w}_{1}\right\|^{2}+\left\|\nabla_{\mathrm{h}} \Lambda^{2 \beta} \boldsymbol{w}_{1}\right\|^{2}+\left\|\Lambda^{2 \beta} \boldsymbol{w}_{1}\right\|^{2}+\left\|\nabla_{\mathrm{h}} \Lambda^{\beta} \boldsymbol{w}_{1}\right\|^{2} \in$ $\mathrm{L}^{1}(0,+\infty)$.

Using the same embeddings as above, we also have that

$$
\begin{aligned}
\tilde{I}_{2} \leq & c_{\varepsilon}\left(\left\|\Lambda^{\beta}\left(\left(\boldsymbol{w}_{2, \mathrm{~h}} \cdot \nabla_{\mathrm{h}}\right) \overline{\boldsymbol{w}}\right)\right\|^{2}+\left\|\Lambda^{\beta}\left(w_{2,3} \partial_{3} \overline{\boldsymbol{w}}\right)\right\|^{2}\right)+\varepsilon\left\|\Lambda^{3 \beta} \overline{\mathrm{u}}\right\|^{2} \\
\leq & \left.c\left(\left\|\Lambda^{\beta} \boldsymbol{w}_{2, \mathrm{~h}}\right\|_{\mathrm{L}^{4}}^{2}\left\|\nabla_{\mathrm{h}} \overline{\boldsymbol{w}}\right\|_{\mathrm{L}^{4}}^{2}+\left\|\boldsymbol{w}_{2, \mathrm{~h}}\right\|_{\mathrm{L}^{\infty}}^{2}\left\|\Lambda^{\beta} \nabla_{\mathrm{h}} \overline{\boldsymbol{w}}\right\|^{2}\right)+\left\|w_{2,3}\right\|_{\mathrm{H}^{1+2 \beta}}^{2}\left\|\partial_{3} \overline{\boldsymbol{w}}\right\|_{\mathrm{H}^{-1+\beta}}^{2}\right) \\
& +\varepsilon \| \Lambda^{3 \beta} \overline{\mathrm{u}}^{2} \\
\leq & c\left\|\Lambda^{2 \beta} \boldsymbol{w}_{2, \mathrm{~h}}\right\|^{2}\left\|\nabla_{\mathrm{h}} \Lambda^{\beta} \overline{\boldsymbol{w}}\right\|^{2}+c\left\|\Lambda^{2 \beta} \boldsymbol{w}_{2, \mathrm{~h}}\right\|^{2}\left\|\Lambda^{\beta} \nabla_{\mathrm{h}} \overline{\boldsymbol{w}}\right\|^{2} \\
& +\left\|\nabla_{\mathrm{h}} \Lambda^{2 \beta} \boldsymbol{w}_{2, \mathrm{~h}}\right\|^{2}\left\|\Lambda^{\beta} \overline{\boldsymbol{w}}\right\|^{2}+\varepsilon\left\|\Lambda^{3 \beta} \overline{\mathrm{u}}\right\|^{2} \\
\leq & C\left(\left\|\nabla_{\mathrm{h}} \Lambda^{\beta} \overline{\mathrm{u}}\right\|^{2}+\left\|\Lambda^{\beta} \overline{\mathrm{u}}\right\|^{2}\right)\left(\left\|\nabla_{\mathrm{h}} \Lambda^{2 \beta} \boldsymbol{w}_{2, \mathrm{~h}}\right\|^{2}+\left\|\Lambda^{2 \beta} \boldsymbol{w}_{2, \mathrm{~h}}\right\|^{2}\right) \\
& +C\left(\left\|\nabla_{\mathrm{h}} \Lambda^{\beta} \overline{\boldsymbol{v}}\right\|^{2}+\left\|\Lambda^{\beta} \overline{\boldsymbol{v}}\right\|^{2}\right)\left(\left\|\nabla_{\mathrm{h}} \Lambda^{2 \beta} \boldsymbol{w}_{2, \mathrm{~h}}\right\|^{2}+\left\|\Lambda^{2 \beta} \boldsymbol{w}_{2, \mathrm{~h}}\right\|^{2}\right)+\varepsilon\left\|\Lambda^{3 \beta} \overline{\mathrm{u}}\right\|^{2}
\end{aligned}
$$

where, in particular we used Lemma 2.2 with $s_{0}=-\beta, s_{1}=1+2 \beta$, and $s_{2}=-1+\beta$. Also in this case $\left\|\nabla_{\mathrm{h}} \Lambda^{2 \beta} \boldsymbol{w}_{2, \mathrm{~h}}\right\|^{2}+\left\|\Lambda^{2 \beta} \boldsymbol{w}_{2, \mathrm{~h}}\right\|^{2} \in \mathrm{L}^{1}(0,+\infty)$.

Therefore, combining (107) along with (108) and (109) (and applying (44)), we get

$$
\begin{aligned}
& \frac{1}{2} \frac{d}{d t}\left(\left\|\Lambda^{2 \beta} \overline{\mathrm{u}}\right\|^{2}+\alpha^{2}\left\|\nabla_{\mathrm{h}} \Lambda^{2 \beta} \overline{\mathrm{u}}\right\|^{2}+\left\|\Lambda^{2 \beta} \bar{\zeta}\right\|_{\mathcal{M}_{\mathrm{h}}^{1}}^{2}\right)+(v-2 \varepsilon)\left(\left\|\Lambda^{3 \beta} \overline{\mathrm{u}}\right\|^{2}+\alpha^{2}\left\|\nabla_{\mathrm{h}} \Lambda^{3 \beta} \overline{\mathrm{u}}\right\|^{2}\right) \\
& \quad+\delta\left\|\Lambda^{2 \beta} \bar{\zeta}\right\|_{\mathcal{M}_{\mathrm{h}}^{1}} \\
& \quad \leq C\left(\left\|\nabla_{\mathrm{h}} \Lambda^{\beta} \overline{\mathrm{u}}\right\|^{2}+\left\|\Lambda^{\beta} \overline{\mathrm{u}}\right\|^{2}\right) \mathbf{d}(t)+C\left(\left\|\nabla_{\mathrm{h}} \Lambda^{\beta} \overline{\boldsymbol{v}}\right\|^{2}+\left\|\Lambda^{\beta} \overline{\boldsymbol{v}}\right\|^{2}\right) \mathbf{d}(t),
\end{aligned}
$$

where $\mathbf{d}(t)=\left\|\Lambda^{3 \beta} \boldsymbol{w}_{1}\right\|^{2}+\sum_{i=1}^{2}\left(\left\|\nabla_{\mathrm{h}} \Lambda^{2 \beta} \boldsymbol{w}_{i}(t)\right\|^{2}+\left\|\Lambda^{2 \beta} \boldsymbol{w}_{i}(t)\right\|^{2}+\left\|\nabla_{\mathrm{h}} \Lambda^{\beta} \boldsymbol{w}_{i}(t)\right\|^{2}\right)$. 
Recalling that $\left(\bar{w}(0), \bar{\eta}^{0}\right)=(0,0)$, and using (105), an application of the Gronwall's lemma produces

$$
\begin{aligned}
& \mathcal{E}_{1+2 \beta}(t)=\left(\left\|\Lambda^{2 \beta} \overline{\mathrm{u}}\right\|^{2}+\alpha^{2}\left\|\nabla_{\mathrm{h}} \Lambda^{2 \beta} \overline{\mathrm{u}}\right\|^{2}+\left\|\Lambda^{2 \beta} \bar{\zeta}\right\|_{\mathcal{M}_{\mathrm{h}}^{1}}^{2}\right)(t) \\
& \leq C \int_{0}^{t} e^{\int_{s}^{t} \mathbf{d}(\ell) d \ell} \mathbf{d}(s)\left(\left\|\nabla_{\mathrm{h}} \Lambda^{\beta} \overline{\boldsymbol{v}}\right\|^{2}+\left\|\Lambda^{\beta} \overline{\boldsymbol{v}}\right\|^{2}\right) d s \\
& \leq C e^{\int_{0}^{t} \mathbf{d}(\ell) d \ell}\left(\int_{0}^{t} \mathbf{d}(s) d s\right) e^{-\varpi t}\left\|U_{0,1}-U_{0,2}\right\|_{\mathcal{H}_{\mathrm{h}}^{1+\beta}} \\
& \leq C e^{\widetilde{C} t}\left\|U_{0,1}-U_{0,2}\right\|_{\mathcal{H}_{\mathrm{h}}^{1+\beta}}
\end{aligned}
$$

where to obtain the final estimate we used the regularity of the solutions $\boldsymbol{w}_{1}$ and $\boldsymbol{w}_{2}$.

The control provided by (110) is exactly the last point of (iv) in Lemma 7.3, and it also concludes the Proof of Theorem 7.2.

Funding Open Access funding provided by Universitá degli Studi di Firenze.

Open Access This article is licensed under a Creative Commons Attribution 4.0 International License, which permits use, sharing, adaptation, distribution and reproduction in any medium or format, as long as you give appropriate credit to the original author(s) and the source, provide a link to the Creative Commons licence, and indicate if changes were made. The images or other third party material in this article are included in the article's Creative Commons licence, unless indicated otherwise in a credit line to the material. If material is not included in the article's Creative Commons licence and your intended use is not permitted by statutory regulation or exceeds the permitted use, you will need to obtain permission directly from the copyright holder. To view a copy of this licence, visit http://creativecommons.org/licenses/by/4.0/.

\section{References}

1. Adams, N.A., Stolz, S.: Deconvolution methods for subgrid-scale approximation in large eddy simulation, In: Geurts, B.J. (Ed.), Modern Simulation Strategies for Turbulent Flow, R.T. Edwards, PA, 2 pp. 1-44 (2001)

2. Ali, H.: Approximate deconvolution model in a bounded domain with vertical regularization. J. Math. Anal. App. 408, 355-363 (2013)

3. Babin, A.V., Vishik, M.I.: Attractors of Evolution Equations. North-Holland, Amsterdam (1992)

4. Berselli, L. C., Iliescu, T., Layton, W.J.: Mathematics of Large Eddy Simulation of Turbulent Flows, In: Chattot, J.J. et al. (eds.) Scientific Computation, Springer, (2006)

5. Berselli, L.C.: Analysis of a Large Eddy simulation model based on anisotropic filtering. J. Math. Anal. Appl. 386, 149-170 (2012)

6. Berselli, L.C., Lewandowski, R.: Convergence of approximate deconvolution models to the mean NavierStokes equations. Ann. Inst. H. Poincaré Anal. Non Linéaire 29, 171-198 (2012)

7. Berselli, L.C., Bisconti, L.: On the structural stability of the Euler-Voigt and Navier-Stokes-Voigt models. Nonlinear Anal. 75, 117-130 (2012)

8. Berselli, L.C., Catania, D.: On the Boussinesq equations with anisotropic filter in a vertical pipe. Dyn. Partial Differ. Equ. 12(2), 177-192 (2015)

9. Berselli, L.C., Catania, D.: On the well-posedness of the Boussinesq equations with anisotropic filter for turbulent flows. Z. Anal. Anwend. 34(1), 61-83 (2015)

10. Berselli, L.C., Catania, D., Lewandowski, R.: Convergence of approximate deconvolution models to the filtered MHD equations. J. Math. Anal. Appl. 401, 864-880 (2013)

11. Bessaih, H., Flandoli, F.: Weak attractor for a dissipative Euler equation. J. Dynam. Diff. Equ. 12(4), 713-732 (2000)

12. Bisconti, L.: On the convergence of an approximate deconvolution model to the $3 \mathrm{D}$ mean Boussinesq equations. Math. Methods Appl. Sci. 38(7), 1437-1450 (2015)

13. Bisconti, L., Catania, D.: Remarks on global attractors for the 3D Navier-Stokes equations with horizontal filtering. Discrete Contin. Dyn. Syst. Ser. B 20(1), 59-75 (2015) 
14. Bisconti, L., Catania, D.: Global well-posedness of the two-dimensional horizontally filtered simplified Bardina turbulence model on a strip-like region. Commun. Pure Appl. Anal. 16(5), 1861-1881 (2017)

15. Catania, D., Secchi, P.: Global existence and finite dimensional global attractor for a 3D double viscous MHD- $\alpha$ model. Commun. Math. Sci. 8(4), 1021-1040 (2010)

16. Conti, M., Danese, V., Giorgi, C., Pata, V.: A model of viscoelasticity with time-dependent memory kernels. Amer. J. Math. 140(2), 349-389 (2018)

17. D'Ancona, P., Foschi, D., Sigmund, S.: Atlas of products for wave-Sobolev spaces on $\mathbb{R}^{1+3}$. Trans. Am. Math. Soc. 364, 31-63 (2012)

18. Deardorff, J.W.: A numerical study of three-dimensional turbulent channel flow at large Reynolds numbers. J. Fluid Mech. 41, 453-480 (1970)

19. Di Plinio, F., Giorgini, A., Pata, V., Temam, R.: Navier-Stokes-Voigt Equations with Memory in 3D Lacking Instantaneous Kinematic Viscosity J. Nonlinear Sci. https://doi.org/10.1007/s00332-017-94221

20. Foias, C., Holm, D., Titi, E.S.: The three dimensional viscous Camassa-Holm equations and their relation to the Navier-Stokes equations and turbulence theory. J. Dynam. Differ. Equ. 14, 1-35 (2002)

21. Gal, C.G., Tachim-Medjo, T.: A Navier-Stokes-Voigt model with memory. Math. Method Appl. Sci. 36, 2507-2523 (2013)

22. Galdi, G.P.: An introduction to the Navier-Stokes initial-boundary value problem, in: Fundamental Directions in Mathematical Fluid Mechanics, In: Galdi, G.P., Heywood, J.G., Rannacher, R. (eds.) Advances in Mathematical Fluid Mechanics, Birkhäuser, Basel, 1-70 (2000)

23. Germano, M.: Differential filters for the large eddy simulation of turbulent flows. Phys. Fluids 29, 17551757 (1986)

24. Grasselli, M., Pata, V.: Uniform attractors of nonautonomous dynamical systems with memory. Evolution equations, semigroups and functional analysis (Milano, 2000). In: Lorenzi, A., Ruf, B. (eds.) Program Nonlinear Differential Equations Applications, vol. 50, Birkhäuser, Basel, pp. 155-178 (2002)

25. Kato, T., Ponce, G.: Commutator estimates and the Euler and Navier-Stokes equations. Commun. Pure Appl. Math. 41, 891-907 (1988)

26. Kenig, C.E., Ponce, G., Vega, L.: Well-posedness of the initial value problem for the Korteweg-de Vries equation. J. Am. Math. Soc. 4, 323-347 (1991)

27. Layton, W.: A simple and stable scale-similarity model for large Eddy simulation: Energy balance and existence of weak solutions. App. Math. Lett. 16(8), 1205-1209 (2003)

28. Lions, J.L., Magenes, E.: Problèmes aux limites non homogènes et Applications. Dunod, Paris (1968)

29. Pata, V., Zucchi, A.: Attractors for a damped hyperbolic equation with linear memory. Adv. Math. Sci. Appl. 11, 505-529 (2001)

30. Sell, G.R.: Global attractor for the three-dimensional Navier-Stokes equations. J. Dynam. Differ. Equ. 8(1), 1-33 (1996)

31. Sell, G.R., You, Y.: Dynamics of s Evolutionary Equations, Applied Mathematical Sciences 143. Springer, Berlin (2002)

32. Selmi, R.: Global well-posedness and convergence results for 3D-regularized Boussinesq system. Canad. J. Math. 64, 1415-1435 (2012)

33. Scotti, A., Meneveau, C., Lilly, D.K.: Generalized Smagorinsky model for anisotropic grids. Phys. Fluids 5, 2306 (1993)

34. Taylor, M.E.: Pseudodifferential Operators and Nonlinear PDE. Birkhäuser Boston Inc., Boston, MA (1991)

35. Temam, R.: Infinite-Dimensional Dynamical Systems in Mechanics and Physics, Applied Mathematical Sciences 68. Springer, Berlin (1997)

36. Wu, J.: Inviscid limits and regularity estimates for the solutions of the 2-D dissipative quasi-geostrophic equations, Indiana Univ. Math. J. 46, 1113-1124 (1997)

Publisher's Note Springer Nature remains neutral with regard to jurisdictional claims in published maps and institutional affiliations. 